

\section{Application of Empirical and Linear Methods to VSTOL Powered-Lift Aerodynamics}

Richard Margason, Ames Research Center, Moffett Field, California

Richard Kuhn, V/STOL Consultant, Valencia, California

February 1988

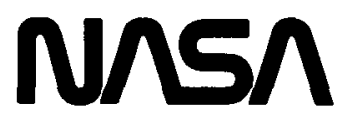

National Aeronautics and

Space Administration

Ames Research Center

Moffett Field, California 94035 


\title{
APPLICATION OF EMPIRICAL AND LINEAR METHODS TO VSTOL POWERED-LIFT AERODYNAMICS
}

\author{
Richard Margason and Richard Kuhn
}

\author{
Ames Research Center
}

ABSTRACT

This paper critically reviews avallable prediction methods and provides an assessment of their strengths and weaknesses. The methods are applied to selected problems which represent the major aero/propulsion interactions for short takeoff and vertical landing (STOVL) aircraft of current interest. The first two problens deal with aerodynamic performance effects during hover: a) out-of-ground effect, and b) in-ground effect. The first problem can be evaluated for some multijet cases; however, the second problem is very difficult to evaluate for multijets. The ground-environment effects due to wall jets and fountain flows directly affect hover performance. In a related problem c) hotgas ingestion affects the engine operation. Both of these problems as well as jet noise affect the ability of people to work near the aircraft and the ability of the aircraft to operate near the ground. Additional problems are d) the power-augmented lift due to jet-flap effects (both in- and out-of-ground effects) and e) the direct jet-lift effects during short takeoff and landing (STOL) operations. The $f$ inal problem is $f$ ) the aerodynamic/propulsion interactions in transition between hover and wing-borne flight. Areas where modern computational fluid dynamics (CFD) methods can provide improvements to the current computational capabilities are identified.

FOR OVER THIRTY YEARS, vertical or short takeoff and landing (VSTOL) aircraft have been developed primarily from experimental investigations because there are many unique aerodynamic/ propulsion-induced effects which occur with these vehicles. These problems are especially significant at low speeds from hover to wing- borne flight. Proper design of VSTOL aircraft requires an accounting of these complex phenomena. Unfortunately, the flows involved are not amenable to purely theoretical predictions at this time. Improved prediction methods are needed to guide experimental programs and to reduce the testing requirements in the future, as well as to provide an understanding of the flow physics. One motivation of this paper is to identify areas where modern computational fluid dynamics (CFD) methods can provide improvements to the current computational capabilities. The ultimate goal would be to develop the ability to completely analyze the aerodynamics throughout the entire flight envelope of an arbitrary USTOL aircraft.

Traditionally, prediction techniques applicable to VSTOL aircraft $(1-8)$ * consist of potential-flow methods with empirically derived corrections and/or adjustments to account for $v$ iscous effects which significantly affect aerodynamic/propulsion interactions. Some of these methods are simple to use but provide results with only limited applicability. Other procedures build on potential-flow-panel methods. The input data tend to be complex and difficult to prepare, and often the results have limited usefulness.

In recent years there has been rapid progress in the development of CFD. This progress is due in part to improvements in digital computers (9) which provide rapid computation speeds (up to hundreds of millions of floatingpoint operations per second), and larger memories (up to hundreds of millions of words). This growth was clearly described in Jameson's survey (10) of the evolution of computational

* Numbers in parentheses designate references at end of paper. 
methods for conventional aerodynamics. Numerical approximation methods were examined for a hierarchy of models in ascending order of complexity, ranging from the linearized potentialflow equations to the Reynolds-averaged NavierStokes equations. In a study (11) of the influence of CFD on experimental aerospace facilities, four main stages (I-IV) of approximation to the full Navier-Stokes equations were identified. For completeness in relation to short takeoff and vertical landing (STOVL) aero/ propulsion interactions, the present authors have added a Stage 0 to represent the empirically based methods, and Stage Ib to represent linearized inviscid methods which include an approximate model for jets:

\begin{tabular}{|c|c|}
\hline Stage & Approximation \\
\hline 0 & Empirical methods \\
\hline I & Linearized inviscid \\
\hline Ia & $\begin{array}{l}\text { Linearized inviscid plus boundary } \\
\text { layer (BL) }\end{array}$ \\
\hline Ib & Linearized inviscid plus modeled Jets \\
\hline I I & Nonlinear inviscid \\
\hline I Ia & Nonlinear inviscid plus interacting BL \\
\hline III & Reynolds-averaged Navier-Stokes \\
\hline IV & $\begin{array}{l}\text { Full Navier-Stokes (large-eddy } \\
\text { simulations with small-scale } \\
\text { turbulence modeling) }\end{array}$ \\
\hline
\end{tabular}

Stage 0 has been the mainstay method for extrapolating experimental data beyond the original wind tunnel test conditions up to the present time for VSTOL aero/propulsion interactions. Stage I is currently used for engineering anay sis of conventional airplanes. The Navy V/STOL Aerodynamics, Stability and Control Manual $(4,5)$ utilizes Stages $0, I$, and Ib methods, and represents the current state of the art in V/STOL computational methods. For conventional airplanes without propulsion-induced effects, limited to moderate use is also being made of stage II solutions; Stage III is expected to be widely used in the next 10 years. The principal items pacing introduction of Stage III into the aerodynamic design process are: a) development of improved turbulence models; b) geometry grid generation difficulties and limitations;

c) development of more efficient and reliable numerical algorithms; and d) development of more powerful scientific computers.

At this time some initial efforts have been made to use stage II and III approximations for
VSTOL. One of the first stage III analyses was by Baker (12). This calculation of the flow field caused by a jet in a crossflow used a finite-element, pressure-interaction, parabolic approximation to the Reynolds-averaged full Navier-Stokes equations. The other papers (13-16) in the present session will present current examples of several Stage II and Stage III approximations applied to either STOL- or STOVL-aircraft-related flow fields.

This paper critically reviews available stage 0 and stage I prediction methods, and attempts to provide an assessment of their strengths and weaknesses. Existing methods will be discussed based on six selected problems which represent the major aero/propulsion interactions for STOVL aircraft of current interest. This paper presents examples using available methods. The first two problems deal with aerodynamic performance effects during hover: a) out-of-ground effect and b) in-ground effect. Both problems are reasonably well understood for the case of a single jet. The first problem can be evaluated for some multijet cases; however, the second problem is very difficult to evaluate for multijets. The next three problems are related to operations near the ground: c) the ground-environment effects due to wall jets and due to surface breakdown, d) hot-gas ingestion effects on the engine operation, and e) short takeoff and landing (STOL) ground effects. These problems affect both the ability of people to work near the aircraft and the ability of the aircraft to operate near the ground. The final problem $f)$ is the aerodynamic/propulsion interactions in transition between hover and wing-borne flight.

\section{NOMENCLATURE}

$\begin{array}{ll}A & \text { jet area } \\ B L & \text { butt line } \\ C_{D} & \text { drag coefficient } \\ C_{L} & \text { lift coefficient } \\ C_{M} & \text { pitching-moment coefficient } \\ C_{T} & \text { thrust coefficient } \\ C_{\mu} & \text { jet momentum coefficient } \\ C_{l} & \text { section lift coefficient } \\ C_{p} & \text { pressure coefficient } \\ c_{\mu}^{\prime} & \text { section jet momentum coefficient } \\ D^{\mu} & \text { plate or jet diameter } \\ d & \text { jet diameter } \\ E V D & \text { elementary vortex distribution } \\ \text { FS } & \text { fuselage station } \\ H, h & \text { height } \\ h_{\mu} & \text { upflow jet thickness } \\ I & \text { turbulent intensity }\end{array}$




\begin{tabular}{|c|c|}
\hline $\mathrm{K}_{\mathrm{h}}$ & $\begin{array}{l}\text { factor for effect of forward speed on } \\
\text { suckdown }\end{array}$ \\
\hline L & lift \\
\hline$\ell$ & stagnation line \\
\hline LID & lift improvement device \\
\hline M & Mach number \\
\hline NPR & nozzle pressure ratio \\
\hline$P, p$ & pressure \\
\hline$q$ & dynamic pressure \\
\hline R & radius, radial distance \\
\hline$R_{n}$ & Reynolds number \\
\hline$s^{\prime \prime}$ & wing area \\
\hline$S^{\prime}$ & $\begin{array}{l}\text { distance between jet impingement points } \\
\text { on a ground plane }\end{array}$ \\
\hline STOL & short takeoff and landing \\
\hline STOVL & short takeoff and vertical landing \\
\hline$T$ & thrust, temperature \\
\hline V & velocity \\
\hline $\begin{array}{l}v_{e} \\
\text { vSTOL } \\
\text { WL }\end{array}$ & $\begin{array}{l}\text { effective velocity ratio, } \sqrt{q / q J} \\
\text { vertical or short takeoff and landing } \\
\text { water line }\end{array}$ \\
\hline$X, Y, Z$ & Cartesian coordinates \\
\hline$\alpha$ & angle-of-attack \\
\hline B & sideslip angle \\
\hline$\Delta L$ & $\begin{array}{l}\text { aero/propulsion lift-interference } \\
\text { increment }\end{array}$ \\
\hline$\Delta M$ & $\begin{array}{l}\text { aero/propulsion lift-interference } \\
\text { increment }\end{array}$ \\
\hline$\Delta \mathrm{T}$ & inlet temperature rise, $T_{i}-T_{0}$ \\
\hline$\delta$ & deflection angle \\
\hline$\delta^{\prime}$ & section deflection angle \\
\hline$\phi$ & velocity potential \\
\hline$\phi^{\prime}$ & $\begin{array}{l}\text { computational polar angle measured in } \\
\text { the ground plane about a jet impinge- } \\
\text { ment point referenced to the line } \\
\text { joining two jet impingement points }\end{array}$ \\
\hline 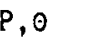 & polar coordinates \\
\hline
\end{tabular}

Subscripts

$\begin{array}{ll}\text { edg } & \text { effective or exit } \\ \text { ej } & \text { jet exit } \\ F & \text { fountain } \\ f & \text { flap or fountain } \\ h & \text { height or hover } \\ \text { i } & \text { inlet or point of maximum rate of change } \\ \text { in } & \text { of decay parameter } \\ j & \text { jet } \\ \text { L } & \text { LID } \\ \text { max } & \text { maximum } \\ n & \text { nozzle } \\ N & \text { normal } \\ 0 & \text { ambient } \\ \text { p1,p2 } & \text { wall jet } \\ t & \text { total } \\ \text { und } & \text { under surface }\end{array}$

$\begin{array}{ll}\text { up } & \text { up from ground plane to undersurface } \\ V & \text { ground vortex } \\ w & \text { wake } \\ x & x \text { direction } \\ \infty & \text { free stream }\end{array}$

\author{
Superseript \\ section \\ MULTI-JET OUT-OF-GROUND EFFECT HOVER
INTERACTIONS
}

During hover, there is a base loss due to interactions between the lifting jets and the lower surface of the aircraft which results in a distribution of induced suction pressures which produce a lift loss. Additional performance losses include inlet-flow distortion, hot-gas ingestion, hot-day conditions, control bleed, internal nozzle flow, thrust vectoring, and static ground effect. There are many items related to the details of the aircraft design which determine the magnitude of the losses. Even though the sum of these losses may be only a few percent of rated thrust, an accurate knowledge of each is required to make a real istic estimate of the aircraft's performance. An error of as little as $3 \%$ in thrust would reduce the gross weight, which in turn would reduce the fuel capacity, and hence the design range, by as much as $10 \%$.

The classic form of an axisymmetric free jet with a uniform nozzle exit dynamic pressure profile and low turbulence is sketched in Fig. 1. In the jet there are two regions of flow: a) the short potential-core region (up to six nozzle diameters long) which has a conical shape and a uniform velocity profile, and b) the fully turbulent region. In an early program intended to evaluate base losses during hover, a NASA investigation (17) evaluated the effect of the character of the jet on induced lift loss. The results showed that a relation exists between the lift loss and the rate of decay of nozzle dynamic pressure.

Both of these parameters are functions of the amount of air entrained into the jet and the proximity of the entrainment to the plate. A correlation between the slope of the lift-loss curve and a parameter indicative of the dynamicpressure decay was developed. As indicated in Fig. 2, this parameter is the maximum slope of dynamic-pressure decay divided by the distance downstream where that slope occurs. The data of (17) were used to obtain the correlation presented in Fig. 2. 


$$
\frac{\Delta L_{w}}{T}=-0.009 \sqrt{\frac{S}{A}} \sqrt{-\frac{\partial q x /\left(P_{n}-p\right)}{\partial x / d_{e}}} \quad\left(\frac{x}{d_{e}}\right)_{i}
$$

where

$S=$ planform area

$A=$ total jet-exit area

$d_{e}=$ diameter of an equivalent single nozzle having an area equal to the total of the areas of the multiple nozzles

$\left(\frac{\partial \mathrm{qx} /\left(\mathrm{P}_{\mathrm{n}}-\mathrm{p}\right)}{\partial \times / \mathrm{d}_{e}}\right)_{\max }=\underset{\text { maximum rate of decay of }}{\text { dynamic pressure }}$

$\left(x / d_{e}\right)_{i}=$ downstream distance at which the dynamic pressure decay rate is maximum

It should be pointed out, however, that the dynamic-decay parameter is quite sensitive to the details of how the data are faired. Since the magnitude of the lift loss is usually a few percent of thrust, a potential estimation error of $10 \%$ amounts to only a few tenths of a percent of the thrust and is not very serious. The solid data symbols in Fig. 2 were obtained from a large-scale test which used a J-85 turbojet engine to provide a hot jet (approximately $1000^{\circ} \mathrm{F}$, and pressure ratios up to 1.73 ). The resultant lift-loss data agree with the correlation derived from small-scale data.

Subsequent investigations by LockheedGeorgia (18), under contract to NASA on configurations derived from the XV-4B airplane, show that this type of correlation can be suitable for additional configurations. However, it was determined that the effect of jet-pressure ratio needed to be included in Eq. 1 by multiplying the right side of the equation by $\left(p_{n} / p\right)-0.64$, and by changing the constant from -0.009 to -0.016 .

A more direct, easier-to-use method for estimating these hover lift losses was developed by McDonnell Aircraft (see Equation in Fig. 33 from (2)). Correlation of data from various single- and multiple-jet configurations resulted in the following expression

$$
\frac{\Delta L_{\infty}}{T}=-0.0002528 \sqrt{\frac{S}{A}}\left[\left(\frac{P_{n}}{p}\right)^{-0.64} \frac{P_{r t}}{d_{e}}\right]^{1.581}
$$

where $P_{r t}$ is the total perimeter of all of the jets in the configuration. It is noted that Eq. 2 implicitly accounts for the higher decay rate of multiple-jet configurations in terms of equivalent jet diameter, but does not account for higher decay rates caused by jet-exit conditions involving high entrainment rates. If higher than normal turbulence levels and decay rates are involved, Eq. 1 should be used.

\section{MULTI-JET IN-GROUND EFFECT HOVER INTERACTIONS}

During hover close to the ground, the lifting jets impinge on the ground and form wall jets flowing radially outward from their impingement points. These outward-flowing wall jets entrain air beneath the configuration and lower the pressure on the bottom surface, causing a jet-induced down load or suckdown. When the wall jets from adjacent jets meet, an upwash or fountain flow is created between them. If there are only two jets, this upwash takes the form of a fan-shaped fountain as shown in Fig. 3. If there are more than two jets, a fanshaped fountain is formed between each adjacent pair of Jets, and a "fountain core" is formed near the centroid of the area enclosed by the jets where the fountain fans from each pair of jets meet. When these fountain flows impinge on the lower surface of the configuration, their momentum imparts an upward force, or lift, that tends to offset the suckdown created by the entrainment action of the wall jets.

The flows involved are highly turbulent and are not amenable to potential-flow analysis. Consequently, the only methods available for estimating their effects on the configuration $(7,19)$ are empirically based. Both methods use essentially the same data base for multiple-jet configurations as does Wyatt's method (20) for estimating the suckdown and Yen's method (21), or a similar approach, for estimating the fountain effects. Wyatt (20) developed an empirical method for estimating the suckdown for singlejet configurations that has become widely accepted. References (7) and (19) use slight modiflcations of Wyatt's method to estimate the suckdown for multiple-jet configurations; reference (19) calculates the suckdown of the individual jets and sums them, and (7) uses Wyatt to calculate the suckdown due to an equivalent 
single fet having the total area and thrust of all the jets of the configuration.

Yen's method (21) for estimating the lift due to the impingement of the fountain basically calculates the flow in the sector of the wall jet from each jet that impinges on the lower surface of the configuration. The momentum of this flow, when it reaches the lower surface of the configuration, is estimated by applying empirically derived factors to account for the velocity decay in the flow along the ground (wall jet) and in the fountain. Unfortunately, the sum of the suckdown based on Wyatt's method, and the fountain lift based on Yen's approach, do not equal the net induced lift for most multijet configurations. In fact, in some cases, as shown in Fig. 4, the twin-jet configuration has more net lift loss than the singlejet configuration. Figure 4 is from (22), which presents data on a twin-jet configuration, and on a single-jet configuration using one of the jets and a planform of half the area of the twin-jet configuration (thus maintaining the same planform-to-jet area ratio). The results indicate that, in addition to the fountain effect, an additional suckdown can be generated on multijet configurations.

In (7) the increment of additional suckdown experienced by multiple jet configurations was extracted from the data base by subtracting the equivalent single-jet suckdown (based on Wyatt) and the fountain increment (based on Yen) from the data. Expressions for estimating this multiple jet suckdown were developed from an empirical correlation of this multiple jet suckdown with the geometric characteristics of the configuration. As shown in Fig. 5, the multijet suckdown term can be larger than the fountain increment. The probable cause of this additional suckdown is shown in Fig. 6 (from (23)). Vortex-like flows are formed between the fountain and each of the adjacent jets. The pressure distributions show that, as expected, the impingement of the fountain flow produces high lifting pressures on the center region of the plate between the jets, but the vortex-like flows between the fountain and the jets induce equally strong suction pressures. The estimated suckdown for a single-jet configuration with the same planform-to-jet-area ratio would correspond to a suction-pressure coefficient, averaged across the area of the plate, about equal to the outer contour line shown in Fig. 7

$\left(C_{p}=-0.004\right)$. Thus, both the 1 ifting pressures and the additional suckdown pressures are much greater than the pressures induced on a singlejet configuration, and the question of whether there is a net lift gain or loss depends on which predominates.

Unfortunately, at the time the methods of $(7,19)$ were developed there were no other pressure data of the type shown in Fig. 7 that could help as a guide in developing the method for estimating multiple-jet effects. More recently similar data (also for a twin-jet configuration) have been published in (24), which show the effects of height (Fig. 7) and jet deflection on these pressure distributions. Additional data of this type are needed to provide a better foundation for estimating the multijet suckdown.

The methods of $(7,19)$ also include factors to account for the effects of lower-surface (fuselage) contour, and lift improvement devices (LIDs) to increase the fountain lift. These factors are based on a very limited data base, and the applicability of the methods to nonplanar configurations is therefore 1 imited. Nevertheless, the methods work well on configurations that fall within the data base. Figure 8 shows good agreement between estimates by the method of $(7)$, and data for a Harrier model (25), with and without LIDs.

On the other hand, Fig. 9 shows a comparison with more recently acquired data (26) for a configuration with a different jet-spacing-tofuselage-width ratio. The method badly overestimates the fountain contribution. There are other shortcomings in the available methods. Reference (26) presents a modification of Wyatt's method for single jets to account for the effects of jet-pressure ratio that appeared to exist in much of the single-jet data base. However, more recent results from a full-scale investigation (27) of the suckdown induced on an 8-foot-diameter plate by the exhaust of a $\mathrm{J}-97$ engine impinging on a ground plane, shows no effect of pressure ratio (Fig. 10). These results also show significantly more suckdown at low heights than was predicted by any of the available methods (Fig. 11). On the other hand, work by Lummus and Smith (22) has shown a small effect of both pressure ratio and jet turbulence (Eig. 12) on a twin-jet configuration.

Before significant improvement can be made in the methods to estimate the suckdown for multiple-jet configurations, it will be necessary to understand and resolve these difficulties in predicting the suckdown for the simple single-jet case. The turbulence measured in (22) was the turbulence in the jet itself. However, it is the wall jet that is entraining air from between itself and the model, and it is probably the turbulence in the wall jet that is important. A significant factor in the turbulence of the jet leaving the model, and probably 
in the turbulence of the wall jet, is the ring vortices generated and traveling downstream in the shear layer of the jet (Fig. 13-photographed by Drubka and Nagib and taken from (28)). It is the growth of these vortices that entrains the surrounding air and causes the jet to decay by transferring energy from the jet to the entrained air, and the transition to turbulence and subsequent jet decay.

Reference (29) has noted that when the jet impinges on the ground, these ring vortices expand outward with the wall jet. They probably play a major role in the entrainment of the air between the model and the ground. A better understanding is needed of the suckdown effects due to jet-induced flows. These flows include the ring vortices, the turbulence of the flow in the jet before it leaves the nozzle, and the wall-jet development and turbulence. A proper modeling of the major features of the flow, including the turbulence in both the free jet and the wall jet, will be required to develop an adequate method for predicting suckdown of single-jet configurations.

Obtaining a better understanding of the flow and an adequate method for predicting the suckdown of single-jet configurations is a necessary first step to developing improved methods for predicting the ground effects on multiple jet configurations. Once these are in hand, the work of Kotansky (2), Saripalli (30), Jenkins and $\mathrm{Hill}$ (31), Kind and Suthanthiran (32), Gilbert (33), Foley and Finley (34), Rizk and Menon (35), and Childs and Nixon (36) on fountain flows can be reexamined.

The $r$ ing vortices created in the shear layer of the free jet (Fig. 13) that expand outward in the wall jet emanating from the impingement point may also be responsible for the extreme unsteadiness of the fountain flow. When these vortices meet at the base of the fountain (as depicted in Fig. 14), their velocity components will not cancel unless they are exactly in phase. The relationship of these vortices to the fountain strength and unsteadiness should be examined. However, the primary need in connection with improving our ability to predict the ground effects on multijet configurations is to obtain a better understanding of the multijet suckdown and the vortex-like flows between the fountain and the jets that apparently generate it (Fig. 6).

\section{HOT GAS INGESTION}

When a jet VTOL configuration hovers in ground effect, the same fountain flows (Fig. 3) that can produce a favorable induced lift can also transport hot gases to the vicinity of an unfavorably placed inlet. The hot gas causes either elevated inlet temperatures or inlet temperature distortion across the engine fan face. Either of these factors will reduce the engine thrust. Alternate engine inlets may be needed to avoid the hot gas. The fountain flows have been the subject of numerous investigations ((30-36) for example). The entrainment, velocity decay, and spreading rates of the wall jet and fountain flows have been studied, and empirical methods for estimating these factors have been determined. Kotansky (2) summarized much of this work and presented the methods developed for estimating the location of the stagnation line on the ground between the jets (Fig. 15), the deflection angle of the upwash fountain if the jets are of unequal thrust, and the momentum of the flow in the fountain.

Green and Zanine (37) have used the same data base to develop a method for estimating the inlet-temperature $r$ ise due to fountain flows. Their method uses the empirically determined velocity and temperature-decay rates developed in the same data base to trace the temperature decay from the impingement point, through the wall jet and fountain, along the lower surface of the configuration to the vicinity of the inlet as depicted in Fig. 16. The method predicts the stagnation lines on the ground and on the under surface of the configuration as shown in Fig. 17. The method adequately predicts the $r$ ise in inlet temperature as the aircraft approaches the ground (Fig. 18), and for some configurations predicts a reversal of temperature rise at the lowest heights that appears to be confirmed by the data.

The method of (37) does not include the effects of LIDs because the data base on these devices is too limited. It also applies only to hover in no-wind conditions. If there is a wind (or in STOL operation) the forward-flowing wall jet will be turned back toward the aircraft in the form of the ground vortex, and this provides another path for hot gases to find their way to the vicinity of the inlet.

The inlet temperature rise is of ten greater at forward speed than at hover. The inlet temperature rises with forward speed (Fig. 19) because, as the velocity increases, the leading edge of the ground-vortex flow field is moved closer to the configuration, and the flow path and time for mixing to reduce the temperature of the gas is shortened. Eventually, a maximum temperature is reached. Finally, as the velocity is increased further, a point is reached where the hot gas cloud is blown below or behind 
the inlet, and no inlet temperature $r$ ise is experienced.

Rudimentary correlations of the maximum inlet temperature $r$ ise experienced are presented in $(38,39)$. For configurations arranged to minimize ingestion, the height of the inlet above the ground was found to be the primary parameter. It was found that the maximum temperature $r$ ise tended to be inversely proportional to the square of the inlet height-todiameter ratio. These results are based on a very small data base and should be used with caution. Most of the data did not go to high enough velocities to establish a correlation of the velocity needed to avoid ingestion, but a method of estimating this speed based on the effect of forward velocity on the depth and forward projection of the ground vortex flow field is presented in (39). Improvements in the ability to estimate inlet temperature rise will depend on developing better methods to predict both the fountain flow fleld and the groundvortex flow field.

JET FLAP IN- AND OUT-OF-GROUND EFFECT

Propulsive lift began in the early days of aviation when the propeller slipstream flowing over the wing was used to attain increased lift. Configuration concepts developed include propeller slipstream, internally blown jet flap, upper surface blown flaps, and externally blown flaps. Along with the concept development and accompanying experimental work $(40,41)$, a number of theoretical and computational methods have been developed to predict the aerodynamic characteristics of these configurations.

The first theoretical method for a wing with powered lift was developed by Spence $(42,43)$. This method gave the 1 ift and pressure distribution on a two-dimensional wing with a jet flap. Spence used a vortex sheet whose vorticity depended on the section momentum coefficient and curvature of the sheet to represent the thin jet wake behind the wing. He obtained the general results shown in Fig. 20 . The lift results in Spence's method are in the form of two equations-one for lift-curve slope and the other for 1 ift due to jet deflection. Both equations contain terms that depend on the section-momentum coefficient. The lift-curve slope, for example, is $(2 \pi)$ modified by two additional terms that account for sectionmomentum coefficient effects. The pressure difference between the upper and lower surface is a function of jet-deflection angle, sectionmomentum coefficient, and chordwise location.
In the pressure coefficient plot in Fig. 20, the computed pressure difference has been combined with the pressure distributions over an ellipse to obtain the total pressure distribution for an elliptical airfoil with a jet flap. This formulation is valid up to a point near the trailing edge as shown in Fig. 20.

Based on Spence's vortex-sheet approxination for the two-dimensional jet wake, a number of three-dimensional methods for jet flaps were then developed. The earliest three-dimensional Jet-flap methods, which included those of Maskell and Spence (44), Hartunian (45), and Das (46), assumed a large aspect ratio and an elliptical spanload distribution. More general three-dimensional jet-flap methods have been developed from methods developed for conventional wings (Fig. 21)-that is, they assumed potential flow and used numerical integration of influence coefficient matrices to obtain a solution. The methods extended conventional theory by including Spence's relationship for the high-momentum jet wake. This implies the assumptions of thin jet sheet and modest wakedeflection angles. Some of these methods include lifting- 1 ine methods by Lissaman (47) and by Lopez and Shen (48) and lifting-surface methods by Goldhammer, Lopez, and Shen (49). In addition, Hackett and Lyman (50) developed an equivalent mechanical-flap method.

Reference (41) presents many comparisons between these theories and experimental data. One example is presented in Fig. 22 for a model with a jet-blown flap over the inboard twothirds of the span, a $45^{\circ}$ flap deflection, and jet-momentum coefficient values ranging from 0.5 to 4.0. The lift, drag, and pitching-moment coefficient results were obtained experimentally using this model, and the corresponding calculated values were obtained using the elementary vortex distribution (EVD) lifting-surface theory (49). The data show generally very good agreement between theory and experiment, especially for the lift and pitching-moment coefficients. It should be noted that the profile drag has not been included in the EVD calculations; adding it would bring the calculated drag into closer agreement with the experimental values.

An example of an application of the jetflap theory to a configuration for which the assumption of a thin jet exhausting near the trailing edge of the wing was violated is presented in Fig. 23. The experimental data, presented in (51), are for an external-blown flap configuration having four engines whose exhausts impinge on a double-slotted flap. Calculations were made for this configuration using experimentally measured distributions of the exhaust 
deflection angle and momentum coefficient at the flap trailing edge (shown on the right in Fig. 23). Such calculations might not be practical in the general situation, of course, since this information would not ordinarily be available. However, this example shows that jet-flap methods can give useful results even though the powered-lift system and resulting flow pattern were more complicated than a simple jet flap. The data show that the maximum and minimim deflection angles occurred on approximately the centerlines of the inboard and outboard engines, respectively, whereas the peak momentum coefficient occurred between the centerlines.

The results on the left in Fig. 23 show that the two jet-flap theories-Lissaman (47) and EVD (49) - give fairly good approximations of lift coefficient for momentum-coefficient values of 0 and 2, but not at a momentum coefficient of 4. The EVD program underpredicted the lift, and the Lissaman program overpredicted it. At least part of this discrepancy could be attributed to the fact that the flap, rather than having a thin jet sheet emanating from its trailing edge, was operating within a relatively thick jet. As a result, noticeable jet interference effects come into play which are not accounted for by the jet-flap theory. Further improvements are needed to deal with complete configurations including all of their complexities. Ultimate solutions for propulsive-lift configurations may require solution the use of Navier-Stokes equations.

JET STOVL CONFIGURATIONS IN STOL OPERATION

The prediction of the aerodynamic forces and moments experienced by jet-and fan-powered STOVL aircraft operating in ground proximity at forward speed (STOL operation) requires modeling a complex set of jet-induced flows in order to cover the entire height and speed range. These induced flows include the flow fields causing the induced lift losses in hover (in- and outof-ground effect), the jet/free-stream interac$t$ ion at forward speed, and the ground vortex created by the interaction of the free stream with the wall jet flowing forward from the jet impingement point. Any method for predicting the aerodynamics of STOVL aircraft in STOL operation must reduce to a method which accounts only for the jet-induced effects at forward speeds.

An empirical method for predicting the effects of ground proximity in STOL operation was developed in (26), and is also available in (6). Reference (26) modifies the method of (7) for hover ground effects to account for the effects of forward speed, and modifies the method of section 2.2.1 of (4) (also in (54)) for the jet-induced effects in transition outof -ground effects to account for the effects of ground proximity. To these is added an empirical method, developed in (26), for estimating the ground-vortex effects, and a fourth term to account for forward-speed effects on the fountain contribution. The terms involved are shown schematically in Fig. 24.

A key factor in the ground effects experienced in STOL operation is the location and strength of the ground vortex (Fig. 25). This ground vortex is formed where the forward flowing wall jet is opposed by the free-stream flow and turned back on itself. This ground vortex induces suction pressures on the ground (and on the lower surface of the aircraft) as shown at the top of Fig. 26. An empirical correlation of the location of the ground vortex was developed in (26) (see also, Fig. 26), and used in the method for estimating the lift loss induced by the ground vortex, and for estimating the effect of forward speed on the hover suckdown term.

As discussed above, and shown in Fig. 24 , the method of (26) for estimating the 1 ift and moment in-ground effect is made up of four terms. The ground-vortex term $(\mathrm{L} / \mathrm{T})_{V}$ and the hover suckdown term $(L / T)_{h}$ induce a negative lift; the jet-wake term $(L / T)_{w}$ also usually induces a lift loss out-of-ground effect, but the proximity of the ground reduces this loss; and the fountain term $(\mathrm{L} / \mathrm{T})_{f}$ produces a favorable or positive lift.

There is a limited data base with which to evaluate the method, and most of it is contained or referenced in (26). Figures 27 (using experimental data from (26)) and 28 (using experimental data from (53)) indicate how well the method reproduces some of the data on which it is based. The estimates of the effects of ground proximity in STOL operation will be most reliable if hover and out-of-ground-effect, jetinduced increments are available on the configuration as a starting point. Additional discussion of pertinent methods may be found in (52).

\section{JET INDUCED EEFECTS IN TRANSITION}

The transition flight regime ranges between hover and wing-borne flight, and uses the combination of jet lift and aerodynamic lift to support the aircraft weight. The lifting jets induce significant changes in the flow field in the vicinity of the aerodynamic lifting surfaces. The interactions between the lift jets 
and the free-stream flow field are due to jet blockage, entrainment, separation in the jet wake, and $l$ ift-induced vortices. These interactions generate a complex flow field which is extremely difficult to analyze.

Existing prediction methods $(2-8)$ usually consist of either empirical procedures or potential-flow methods with empirical corrections to account for many features of the liftjet/free-stream interactions 1 isted in the previous paragraph. The empirical procedures tend to be easier to use and are often used in the preliminary design stage to identify the configurations which are the most appropriate for further design effort. The latter group of methods tend to require input data which are very time-consuming to prepare, and can require large amounts of computer time. As a result, they may be used for more detailed configuration evaluation; however, at their present state of development they are not consistently reliable nor are they adequately validated, and they cannot be counted on to provide more accurate results than the empirical methods.

The most commonly used empirical method for estimating the jet-induced effects on VSTOL configurations in the transition flight regime is the one developed by Kuhn (in section 2.2.2 of (4), and also in (54)). A less general version of this method which is suitable for a simplified preliminary design was presented as a computer program with a listing and sample input and output data (5). In either version of this method, its application is limited to configurations which are consistent with the data base used to develop the method. The approach used in this preliminary design method is outlined in the following paragraph.

First, the pressure coefficient data obtained by Fearn $(55,56)$ in an experimental investigation were used to develop the empirical formulation. These data were obtained for effective velocity ratios $\left(V_{e}\right)$ ranging from 0.10 to 0.45 in an experiment which had a 4-inchdiameter jet exhausting normally to the free stream, and from a large flat plate. The data were presented in pressure coefficients which represent the jet-induced increment. Equations were fit to represent the measured pressure distributions as a function of the effective velocity ratio, as well as the longitudinal and lateral distances from the jet exit. Next, these relations were integrated over the planform of the configuration of interest relative to the nozzle locations to estimate the jetinduced aerodynamics in transition flight. The results were then compared with available experimental data for configurations which ranged from simple flat plates (Figs. 29 and 30 ) to more realistic aircraft configurations

(Fig. 31). These comparisons provided a basis for refining the estimates to account for configuration variables such as jet-nozzle locatic relative to the wing and fuselage, planformto-jet exit area, jet deflection angle, and fuselage lower surface contour. These comparisons also identified limitations of the metrod (Fig. 32). Configurations with a jet located near the wing or other lifting-surface trailing edge have additional jet-flap-like induced lift which is inadequately accounted for by this method. These effects were discussed earlier in the section entitled "Jet Flap In- and Out-ofGround Effect," and may be accounted for by any of the several extensions of Spence's jet-flap theory $(40-47)$.

Several potential-flow, vortex-lattice or panel-method-based schemes have beer developed which account for jet-induced interactions. A comparison of five production-surface panel methods (57) demonstrated that good agreement for conventional aircraft aerodynamics can be achieved between panel-method results and experimental data. One of the earliest comprehensive methods which used a vortex-lattice aircraft model (58) and a source-doublet jet model was developed by Wooler $(1,59)$. This method provided good comparisons between calculated and experimental results for a limited number of VSTOL aircraft configurations. Examples of other methods have shown useful results $(60-64)$.

The complexity of predicting propulsiveinduced effects on VSTOL aircraft prompted Wooler to use a modular approach (Fig. 33). The jet model is incompressible and neglects viscous effects other than the entrainment caused by potential flow sinks or doublets. The entrainment of free-stream flow into the jet and the pressure force on the jet boundary govern the equations of motion of the jet. The entrainment parameters are obtained from experimental data. The variation of the jet cross section is established, based on experimental observations, by a circular jet transforming into an ellipse. The induced-velocity flow field due to the jet interference is obtained from two singularities: a) a uniform sink distribution on axes normal to the free stream at discrete locations along the jet centerline represent the entrained flow, and b) a doublet distribution along the jet centerline represents the blockage and jet-induced circulation effects. Additional procedures are developed to represent nonaxisymmetric jets, jet pairs, and a jet in a nonuniform free stream. 
Another module evaluates the jet-induced forces and moments using a version of the vortex-lattice method from (58) to represent ifting planforms. The method also gives good agreement with experimental data for wing-body combinations by including the planform of the body in addition to the wing. Power-induced aerodynamic characteristics are evaluated by using a propulsion-induced camber distribution on the planform to satisfy the flow-tangency condition at the three-quarter chord of the horseshoe vortices of the lattice representation. This wing-body program was used to improve the method of (1), which represented only the wing.

Experimental data $(65,66)$ from the model shown in Fig. 34 were used to evaluate these methods. These data were obtained in conjunction with the research program (1). Using the method of (1), Mineck (67) obtained good agreement with experimental data from the configuration with the nozzles in the aft position (Fig. 35). For this case the jet-induced increase in wing lift (i.e., jet-flap effect) dominates. In contrast, poor agreement was obtained with the nozzles in the forward position (Fig. 35). The improved wooler method of (59) was also used to evaluate these cases (Fig. 36). The wing-alone calculation based on the original method provided poor agreement for the forward jet position. To represent the presence of the large nacelles and the body, a vortex-lattice method (58) was used to include them in the planform for the improved Wooler method. The improved correlation is shown in Fig. 36. These results indicate that this improved method (59) by Wooler is suitable for propulsion-induced aerodynamic forces and moments at the conceptual and preliminary design stage. The output from the jet-induced flowfield module can be used with a panel method for more detailed analysis.

In an early application of panel methods to model V/STOL aero/propulsion interactions, Rubbert (68) used the Boeing TEA-230 panel method to represent a jet in a crossflow. The specification of the Neumann jet-boundary conditions proved to be difficult because the detailed distribution of jet entrainment around the curved, three dimensional lifting jet was unknown. A later attempt by Siclari (64) at Grumman was more successful. This method used empirical and theoretical data that describe the development of free jets to specify the needed jet-boundary conditions. A set of mixing data was developed which simulated a) the viscous entrainment of ambient air for both free jets and wall jets; b) the free-jet shape in cross-flow to provide the jet-blocking effect; and c) the jet formation along the ground which interacts with other jets leading to the "fountain" effect.

These jet data were combined with an existing wing-body panel method (Fig. 37). The usual zero-normal-velocity boundary was used for the aircraft; the normal inflow velocity, simulating mass entrainment, was used for the jet and ground plane. In ground effect, the fountain momentum and incidence are calculated separately, with empirical data used to calculate additional fountain forces and moments. One correlation with test data from the VAK-191 VTOL aircraft is presented in Fig. 38 for 1 ift and pitching moment coefficients. These data are for a lift engine thrust deflection of $77.5^{\circ}$ and a lift/cruise thrust deflection of $60^{\circ}$ at an effective velocity ratio of approximately 0.14 . At an angle-of-attack of $0^{\circ}$, the computed power-off results agree well with the experimental data, and the computed power-on liftcoefficient results agree well with experimental data. As in the computed results from Wooler's original method by Mineck (67), the poor agreement for power-on pitching moment indicates a need to include a separated-flow representation in the wake portion of the jet.

A further indication of the need for a method which accounts for the lifting-jet-wakeinduced separation is shown in Fig. 39 from a $V / S T O L$ aircraft transition flight analysis (69). This analysis used the PANAIR panel method to represent the aircraft with Neumann boundary condition and a parabolized NavierStokes solution for the lifting jet with an entrainment boundary condition. The jet shape and entrainment were determined using the AdlerBaron jet-in-a-crossflow method (70) and recent unpublished experimental data acquired by Adler. The method was applied to a simple highwing aircraft configuration with a single lifting jet. Unfortunately, the calculated results (Eig. 39) using this analysis do not account for the separated wake due to the lifting jet. As a result, the propulsion-induced lift loss is underestimated by about $20 \%$ of the thrust.

\section{CONCLUDING REMARKS}

For over 30 years, VSTOL aircraft have been developed primarily from experimental investigations because there are many unique aerodynamic/ propulsion-induced effects which occur with these vehicles. The existing data base has been used to develop some empirical and modifiedlinear, potential-flow methods for predicting 
the induced aerodynamic effects. The adequacy of available methods is graphically portrayed in Fig. 40. For propulsion induced effects incurred out-of-ground effect, available methods are adequate in hover and moderately adequate for transition flight. For propulsion-induced effects incurred in-ground effect the interactions are more complicated and available methods are inadequate.

Improved prediction methods are needed to guide experimental programs and to reduce the testing requirements in the future, as well as to provide an understanding of the flow physics. The Navy V/STOL Aerodynamics, Stability and Control Manual $(4,5)$ utilizes empirical methods, linear-inviscid methods, and linearinviscid plus boundary-layer methods, and represents the current state of the art in $\mathrm{V} / \mathrm{STOL}$ computational methods. These methods are very useful for preliminary design of configurations which are consistent with the underlying empirical data base. These methods are usually not suitable for detalled aircraft design. Multiple jet aircraft out-of-ground effect hover interactions represent a lift loss of a few percent of the total thrust, and these interactions may be estimated adequately by empirical procedures to within a percent of the total thrust. In ground effect, the lift-jet/ ground interaction creates complex near-field fountain flows which are difficult to analyze for induced forces and moments and for hot-gas ingestion. Jet-flap theories provide useful preliminary design estimates, but they do not account for all of the major jet interference effects. For lifting jet-induced effects in transition flight, the empirical methods of Kuhn (54) are limited to configurations which are consistent with the data base used to develop the methods.

While existing empirical methods are inadequate for detailed aircraft design, results from current applications of Reynolds-averaged Navier-Stokes solutions $(12-16,35,36)$ offer a promising approach. Initial parabolic NavierStokes applications $(12,69)$ to STOVL aircraft configurations do not adequately handle jetwake-induced flow-separation effects. While initial elliptic Navier-Stokes solutions are demonstrating greatly improved results, there is a need for improved turbulence models for the propulsion-dominated flows. Based on current CFD activity, the Reynolds-averaged NavierStokes solutions should achieve major improvements in the next several years and lead to analysis methods which can estimate aero/ propulsion interactions in a manner suitable for detailed aircraft design applications.

\section{REFERENCES}

1. Wooler, P. T.; Burghart, G. H.; and Gallagher, J. T.: V/STOL Aircraft Aerodynamic Prediction Methods: Vol I - Theoretical Developments of Prediction Methods; vol.II Application of Prediction Methods; Vol. Ii. Manual for Computer Programs; and Vol. IV Literature Survey. AFFDL TR-72-26, January 1972.

2. Kotansky, D. R.: Jet Flowfields. AGARD R-710, 7.1-7.48, 1984.

3. Kuhn, R. E.: An Engineering Method for Estimating the Lateral/Directional

Characteristics of V/STOL Configurations in Transition. Rpt. No. NADC-81031-60, February 1981.

4. Henderson, C.; Clark, J.; and Walters, M.: V/STOL Aerodynamics, Stability and Control Manual. Rpt. No. NADC 80017-60, January 1980.

5. Walters, Marvin M.; and Palmer, Robert E.: Method for Predicting the Jet-Induced Aerodynamics of V/STOL Configurations in Transition. Rpt. No. NADC-80025-60, January 1981.

6. Stewart, V. R.; and Kuhn, R. E.: A Method for Predicting the Aerodynamic Stability and Control Parameters of STOL Aircraft Configurations. AFWAL TR-87-3019, June 1987.

7. Kuhn, R. E.: An Engineering Method for Estimating the Induced Lift on V/STOL Aircraft Hovering in and out of Ground Effect. Naval Air Development Center, Warminster PA, NADC-80246-60, January 1981.

8. Platzer, Max F.; and Margason, Richard J.: Prediction Methods for Jet V/STOL Propulsion Aerodynamics. J. Aircraft, Vol.15, No. 2, 69-77, February, 1978.

9. Peterson, V. L.: Impact of Computers on Aerodynamics Research and Development. Proceedings of the IEEE, Vol. 72, No. 1, 68-79, January 1984.

10. Jameson, A.: The Evolution of Computational Methods in Aerodynamics. Transactions of the ASME, Vol. 50, 1052-1070, December 1983.

11. The Influence of Computational Fluid Dynamics on Experimental Aerospace Facilities, a fifteen year projection prepared by the Comittee on Computational Aerodynamics Simulation Technology Developments, National Research Council, National Academy Press, Washington, DC, 1983. 
12. Baker, A. J.; and Orzechowski, J. A.: Prediction of Turbulent Near-Field Evolution of a Jet in Crossflow Using a PNS Solver. Rpt. No. NADC-86076-60, January 1986.

13. Takanashi, Susumu; and Sawada, Keisuke: Numerical Simulation of Compressible Elow Field about Complete ASKA Aircraft Configuration. SAE paper 872346 , International Powered-Lift Conference, December 1987.

14. Roth, Karlin R.: Numerical Simulation of a Subsonic Jet in a Crossflow. SAE Paper 372343, International Powered-Lift Conference, December 1987.

15. VanDalsem, H. R.; Panaras, A. G.; Rao, K. V.; and Steger, J. L.: Numerical Investigation of Single and Multiple Jets in Ground Effect. SAE Paper 872344, International Powered-Lift Conference, December 1987.

16. Childs, Robert $\varepsilon$.; and Nixon, David: Study of Turbulence and Fluid/Acoustic Interaction in Impinging Jets. SAE Paper 872345, International Powered-Lift Conference, December 1987.

17. Gentry, Garl L.; and Margason, Richard J.: Jet-Induced Lift Losses on VTOL Configurations Hovering In and Out of Ground Effect. NASA TN D-3166, February 1966.

18. Shumpert, P. K.; and Tibbetts, J. G.: Model Tests of Jet-Induced Lift Effects on a VTOL Aircraft in Hover. NASA CR-1297, March 1969.

19. Foley, W. H.; and Sansone, J. A.: V/STOL Propulsion-Induced Aerodynamic Hover Calculations Method. Naval Air Development Center, Warminster PA, NADC-78242-60, February 1980.

20. Wyatt, L. A.: Static Tests of Ground Effect on Planforms Fitted with a CentrallyLocated Round Lifting Jet. Ministry of Avlation CP 749, June 1962.

21. Yen, K. T.: On the Vertical Momentum of the Fountain Produced by Multi-Jet Vertical Impingement on a Flat Ground Plane. Naval Air Development Center, Warminster PA, NADC-79273-60, November 1979.

22. Lummus, J. R. and Smith. E. W.: Flowfield Characteristics and the Effect of Jet-Exhaust Simulation for V/STOL Vehicles Near the Ground. Proceedings of the NADC V/STOL Aircraft Aerodynamic Symposium, Naval Postgraduate School, Monterey, CA, 293-313, May 1979.

23. Hall, G. R.; and Rogers, K. H.: Recirculation Effects Produced by a Pair of Heated Jets Impinging on a Ground Plane. NASA CR 1307, May 1969.
24. Dudley, M. R.; Eshleman, J. E.; and Schell, C. J.: Full-Scale Ground Effects of Twin Impinging Jets Beneath a Subsonic Tactical V/STOL Aircraft. AIAA Paper 86-2704, October 1986.

25. Johnson, D. B.; Lacey, T. R.; and Voda, J. J.: Powered Wind Tunnel Testing of the AV-8B; A Straightforward Approach Pays off. AIAA Paper 79-0333, January 1979.

26. Stewart, V. R.; and Kuhn, R. E.: A Method for Estimating the Propulsion Induced Aerodynamic Characteristics of STOL Aircraft in Ground Effect. NADC-80226-60, August 1983.

27. Christiansen, R. S.: A Large Scale Investigation of VSTOL Ground Effects. AIAA Paper 84-0336, January 1984.

28. VanDyke, M.: An Album of Eluid Motion. The Parabolic Press, Stanford, CA, 1982.

29. Cimbala, J. M.; Stinebring, D. R.; Treaster, A. L.; and Billet, M. L.: Experimental Investigation of a Jet Impinging on a Ground Plane in the Presence of a Cross Flow.

NADC-87019-60, March 1987.

30. Saripalli, K. R.: Laser Doppler Velocimeter Measurements in a 3-D Impinging Twin-Jet Fountain Flow. NASA CP-2462, 147-160, August 1985.

31. Jenkins, R. C.; and Hill, W. G. Jr.: Investigation of VTOL Upwash Flows Formed by Two Impinging Jets. Grumman Research Dept., Rpt. No. RE-548, November 1977.

32. Kind, R. J.; and Suthanthiran: The Interaction of Two Opposing Plane Turbulent Wall Jets. AIAA Paper 72-211, January 1972.

33. Gilbert, B. L.: An Investigation of Turbulence Mechanisms in V/STOL Upwash Flow Fields. Grumman Aerospace, Rpt. No. RE-688, 1984.

34. Foley, W. H.; and Finley, D. B.: Fountain Jet Turbulence. AIAA Paper 81-1293, June 1981.

35. Rizk, M. H.; and Menon, S.: Numerical Investigation of $\mathrm{V} / \mathrm{STOL}$ Jet Induced Interactions. NASA CP-2462, 161-194, August 1985.

36. Childs, R. E.; and Nixon, D.: Unsteady Three-Dimensional Simulations of VTOL Upwash Fountain Turbulence. NASA CP-2462, 195-206, August 1985.

37. Green, K. A.; and Zanine, J. J.: Estimation of Hot Gas Reingestion for a VTOL Aircraft at the Conceptual Design Stage. SAE Paper SP-591, V/STOL: an Update and Overview, 51-66, October 1984.

38. Kuhn, R. E.: Design Concepts for Minimizing Hot-Gas Ingestion in V/STOL Aircraft. AIAA Paper 81-1624, August 1981. 
39. Kuhn, R. E.: Hot Gas Ingestion and the Speed Needed to Avoid Ingestion for Transport Type STO/VL and STOL Configurations", AIAA Paper 84-2530, October 1984.

40. Lowry, John G.; Riebe, John M.: and Campbel1, John P.: The Jet-Augmented Flap. Preprint No. 715, S.M.F. Fund Paper, Inst. Aeronaut. Sci., January 1957.

41. Margason, Richard J.; Yip, Long P.; and Gainer, Thomas G.: Recent Developments in Propulsive-Lift Aerodynamic Theory. Aerodynamic Anayses Requiring Advanced Computers, Part I, NASA SP-347, 543-565, 1975.

42. Spence, D. A.: The Lift Coefficient of a Thin, Jet-Flapped Wing. Proc. Roy. Soc. (London), Ser. A, Vol. 238, No. 1212, 46-68, December 4, 1956.

43. Spence, D. A.: Some Simple Results for Two-Dimensional Jet-Flap Aerofoils. Aeronaut. Quart., Vol. IX, Pt. IV, 395-406, November 1958.

44. Maskel1, E. C.; and Spence, D. A.: A Theory of the Jet Flap in Three Dimensions. Proc. Roy. Soc. (London), Ser. A, Vol. 251, No. 1266, 406-425, June 9, 1959.

45. Hartunian, Richard A.: The Einite Aspect Ratio Jet Flap. Rep. No. A1-1190-A-3 (Contract No. DA 44-177-TC-439), Cornell Aeronaut. Lab., Inc., October 1959.

46. Das, A.: Theoretical and Experimental Testing on Jet Flap Wings. Part I: Testing of a Rectangular Wing at Various Aspect Ratios. NASA TT E-13715, 1971.

47. Lissaman, Peter B. S.: Analysis of High-Aspect-Ratio Jet-Flap Wings of Arbitrary Geometry. NASA CR-2179, 1973.

48. Lopez, M. L.; and Shen, C. C.: Recent Developments in Jet Flap Theory and Its Application to STOL Aerodynamic Analysis. AIAA Paper 71-578, June 1971.

49. Goldhammer, M. I.; Lopez, M. L.; and Shen, C. C.: Methods for Predicting the Aerodynamic and Stability and Control Characteristics of STOL Aircraft.

Volume I - Basic Theoretical Methods. AFFDL-TR-73-146, Vol. I, U. S. Air Force, December 1973.

50. Hackett, J. E.; and Lyman, V.: The Jet Flap in Three Dimensions: Theory and Experiment. AIAA Paper 73-653, July 1973.

51. Johnson, William G., Jr.; and Kardas, Gerald E.: A Wind-Tunnel Investigation of the Wake Near the Trailing Edge of a Deflected Externally Blown Flap. NASA TN X-3079, 1974. 52. Ransom, E. C. P.; and Smy, J. R.: Introduction and Review of Some Jet Interference Phenomena Relevant to $\mathrm{V} / \mathrm{STOL}$ Aircraft. AGARD $R-710,2.1-2.23,1984$.
53. Margason, R. J.; Vogler, R. D. ; and Winston, M. M.: Wind Tunnel Investigation at Low Speed of a Model of the Kestrel $(X V-6 A)$ Vectored Thrust V/STOL Airplane. NASA TN D-6826, July 1972.

54. Kuhn, R. E.: An Empirical Method for Estimating the Jet-Induced Effects on V/ST? Configurations in Transition. Rpt.

No. R-AMPAC-113, Aerospace Mass Properties Analysis, Inc., November 1979.

55. Fearn, R. L.; and Weston, R. P.: Induced Pressure Distribution of a Jet In a Crossflow. NASA TN D-7916, July 1975.

56. Fearn, R. L.; Kalota, C. ; and Dietz, W. E., Jr.: A Jet/Aerodynamic Surface Interference Model. Proceedings NADC V/STOL Aircraft Aerodynamics, May 1979.

57. Margason, R. J.; Kjelgaard, S. 0.; Sellers, W. L., III; Morris, C. E. K., Jr.; Walkey, K. B.; and Shields, E. W.: Subsonic Panel Methods - A Comparison of Several Production Codes. AIAA Paper 85-0280, January 1985.

58. Margason, R. J.; and Lamar, J. E. : Vortex-Lattice FORTRAN Program for Estimating Subsonic Aerodynamic Characteristics of Complex Planforms. NASA TN D-6142, Eebruary 1971.

59. Wooler, P. T.: "Propulsion - Induced Effects on a Supersonic V/STOL Fighter/Attack Aircraft. Proceedings NADC V/STOL Aircraft Aerodynamics, May 1979.

60. Beatty, T. D.: A Prediction Methodology for Propulsive Induced Forces and Moments in Transition and STOL Flight. Proceedings NADC V/STOL Aircraft Aerodynamics, May 1979.

61. Knott, P. G.: A Review of Some Fundamentals of Lifting Jet Interference with Particular Reference to U.S. Navy Type A and B Concepts. Proceedings NADC V/STOL Aircraft Aerodynamics, May 1979.

62. Perkins, S. C.; and Mendenhall, M. R.: Surface Pressure Distribution on a Flat Plate or Body of Revolution from which a Jet is Issuing. Proceedings NADC V/STOL Aircraft Aerodynamics, May 1979.

63. McMahon, H.: Flap Surface Pressures Behind a Jet Issuing from a Wing in Crossflow. Proceedings NADC V/STOL Aircraft Aerodynamics, May 1979.

64. Siclari, M.; Migdal, D.; and Palcza, J. L.: Development of Theoretical Models for Jet Induced Effects on V/STOL Aircraft. Proceedings NADC V/STOL Aircraft Aerodynamics, May 1979 .

65. Mineck, R. E.; and Schwendemann, M. F.: Aerodynamic Characteristics of a Vectored-Thrust V/STOL Fighter in the Transition-Speed Range. NASA TN D-7191, 1973. 
66. Mineck, R. E. ; and Margason, R. J.: Pressure Distribution on a Vectored-Thrust $\mathrm{V} / \mathrm{STOL}$ Fighter in the Transition-Speed Range. NASA TM X-2867, 1974.

67. Mineck, R. E.: Comparison of Theoretical and Experimental Interference Effects on a Jet VTOL Airplane Model. Proceedings NAVAIR Prediction Methods for Jet V/STOL Propulsion Aerodynamics, July 1975.

68. Rubbert, P. E.: Calculation of Jet Interference Effects on V/STOL Aircraft by a Nonplanar Potential Flow Method. Proceedings Analysis of a Jet in a Subsonic Crosswind, NASA SP-218, 181-204, September 1969.

69. Howell, G. A.: Automated Surface and Plume Simulation Procedure for Use with Aerodynamic Panel Codes. NASA CR 177420, May 1986.
70. Foley, W. H.; and Sansone, J. A.: V/STOL Propulsion-Induced Aerodynamic Hover Calculation Method. NADC-78242-60, February 1980.

71. Bore, C. L.: Ground Based Testing Without Wind Tunnels. ACARD-R710, 10.1-10.6, 1984.

72. Siclari, M. J.; Barche, J.; and Migdal, D.: V/STOL Aircraft Prediction Technique Development for Jet-Induced Effects. NAPTC Report No. PDR-623-18, April 1975.

73. Kuhn, R. E.; DelFrate, J.H.; and Eshleman, J. E.: Ground Vortex Flow Field Investigation. NASA CP (ground vortex workshop), April 1987.

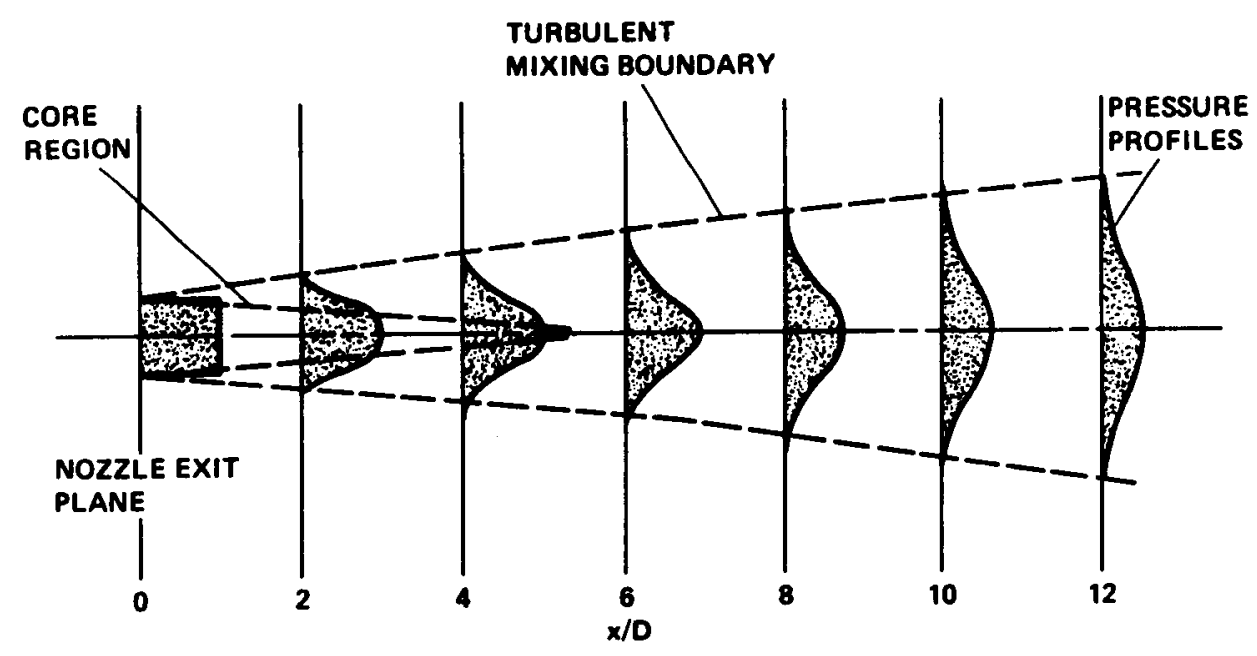

Fig. 1. Schematic of the decay and spread of the jet efflux with distance downstream from the nozzle exit. 


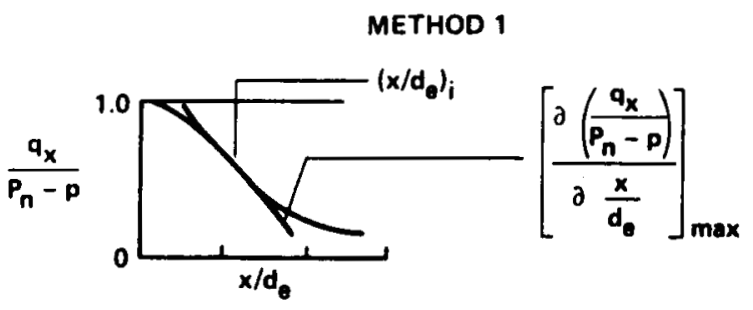

(a)

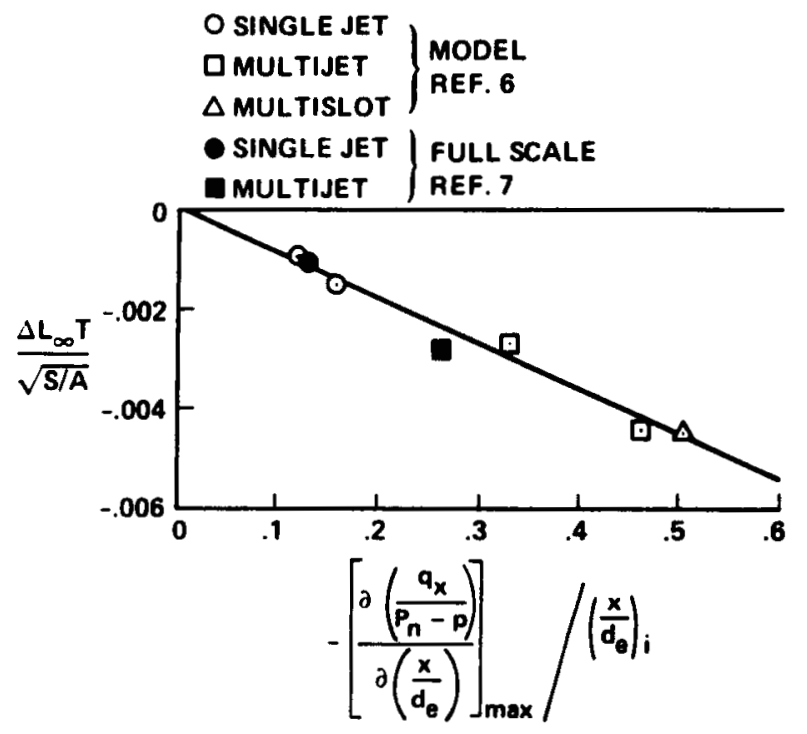

(b)

Fig. 2. Correlation of induced loads with jet decay parameter $\left(p_{t, p} / p=2.0\right)$.

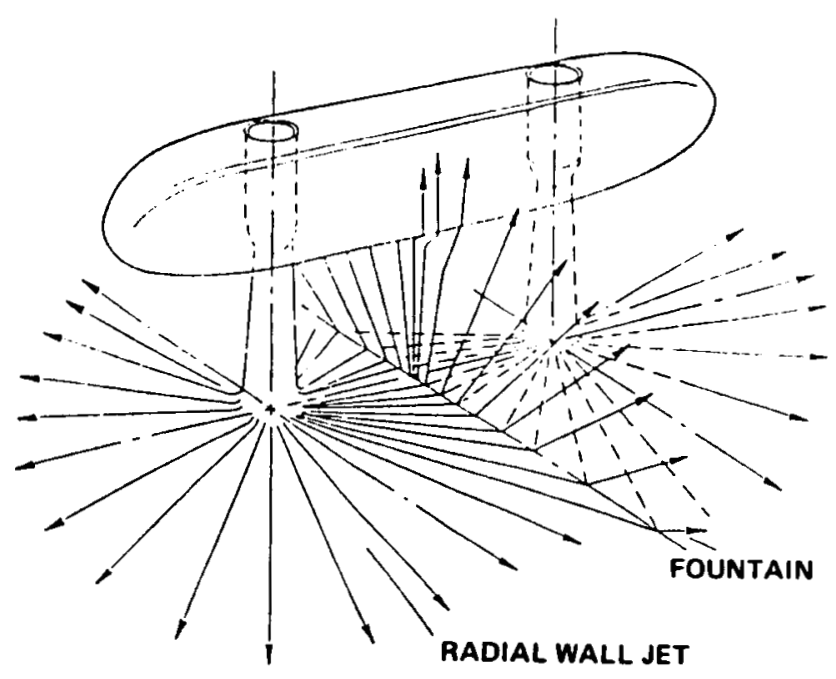

Fig. 3. Fountain flow developed between a pair of lifting jets.

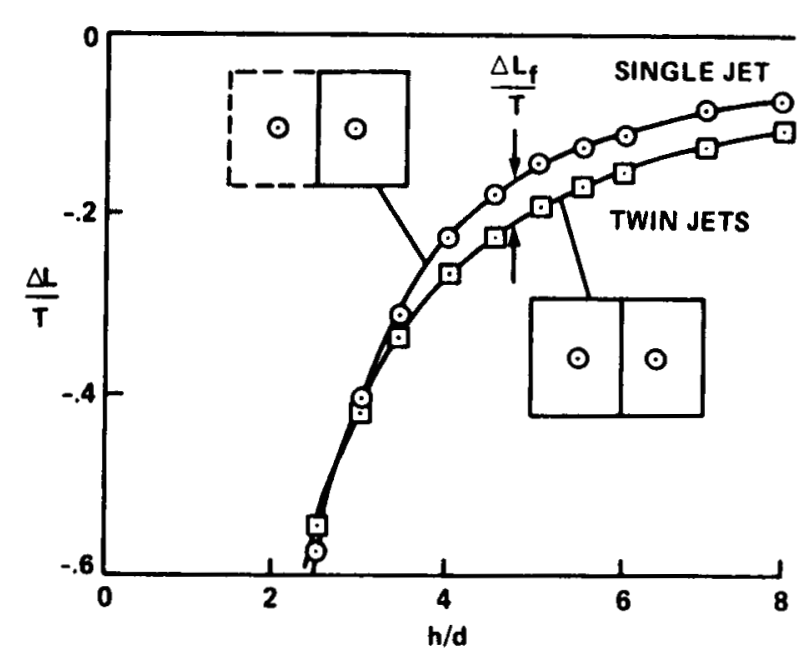

Fig. 4. Some multiple-jet configurations can experience more suckdown than an equivalent single-jet configuration (22). 


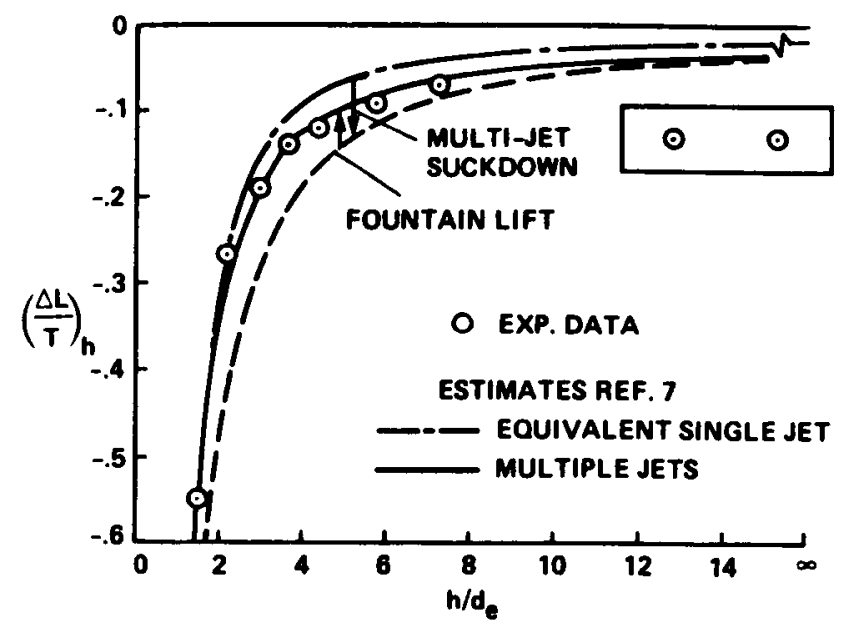

Fig. 5. Fountain 1 ift and the additional multiple-jet suckdown generated on a simple twinjet configuration $(7)$.
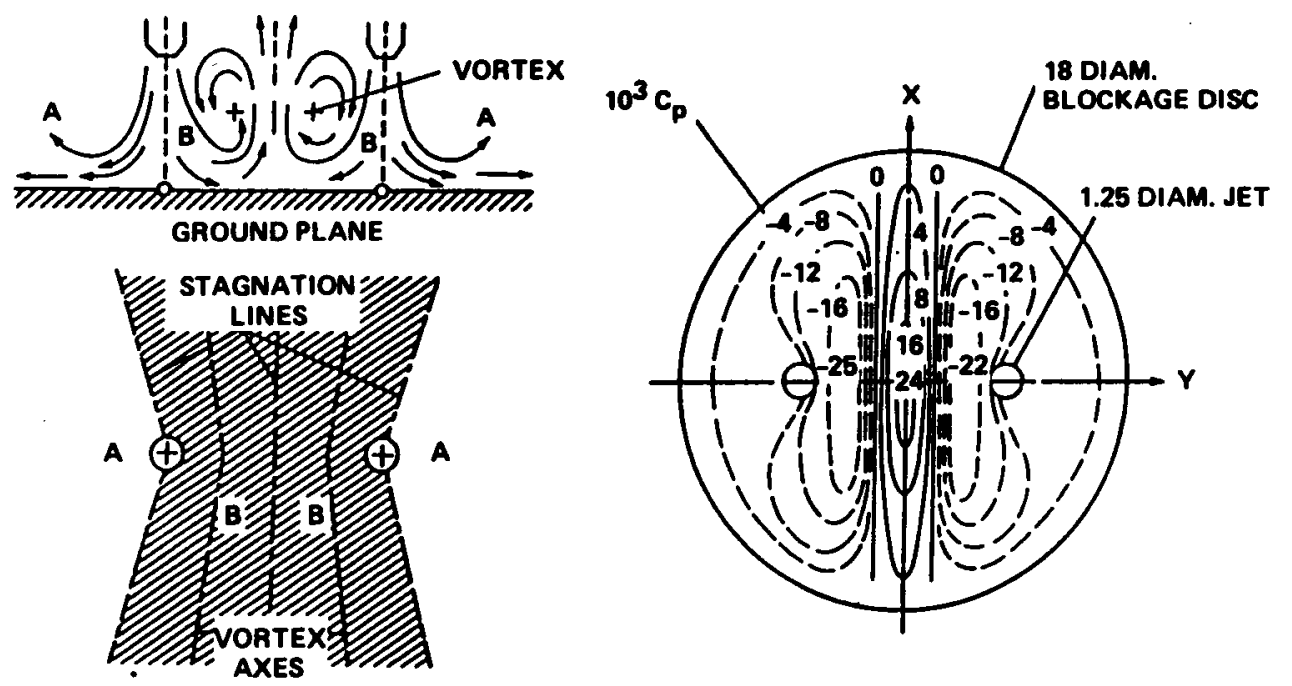

Fig. 6. Flow field and induced pressure distribution for a twin-jet configuration (23). 


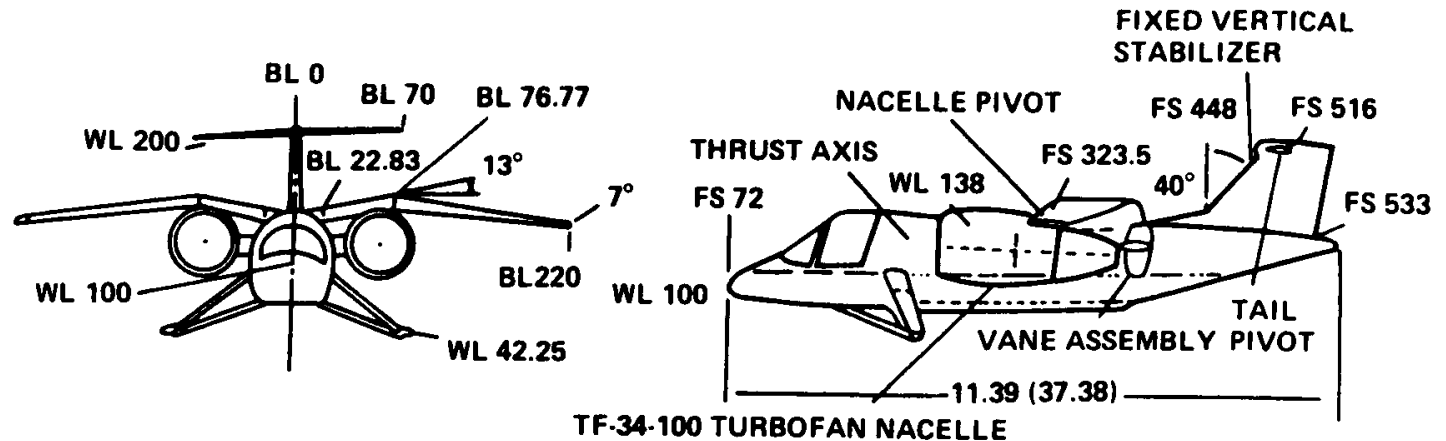

TF-34-100 TURBOFAN NACELLE

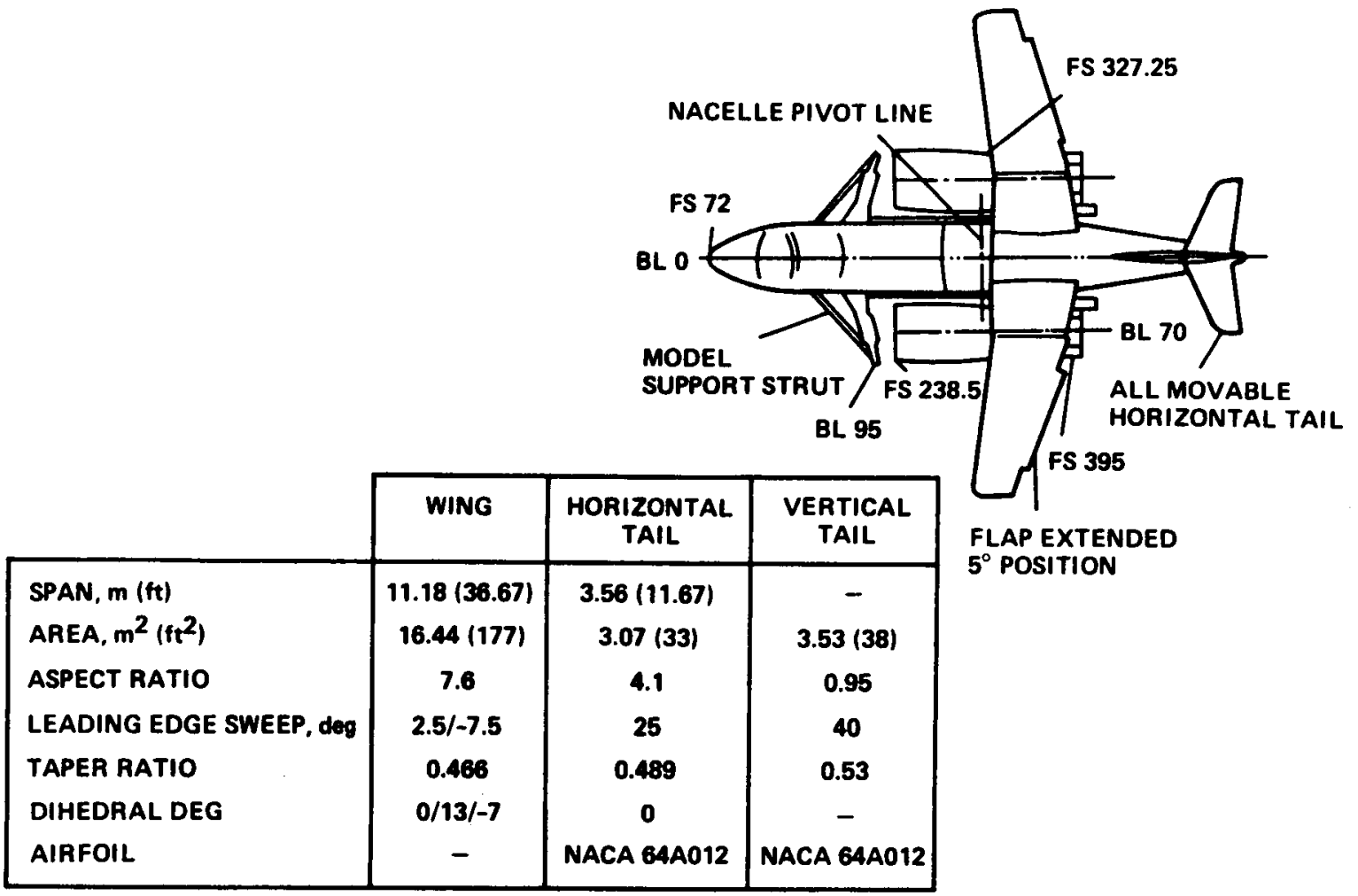

a)
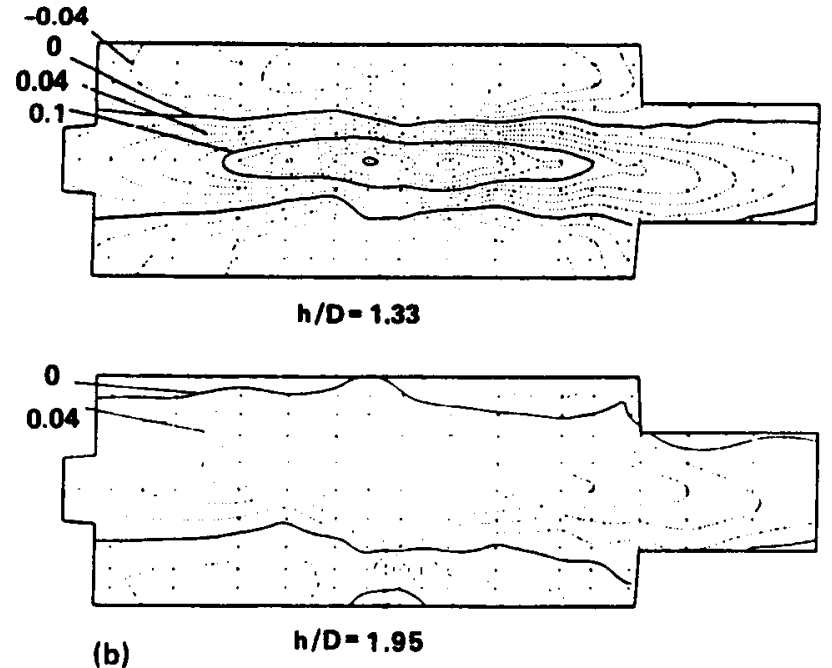

Fig. 7. Three-view configuration and geometry (a) and pressure contours (b) of a twin-jet configuration (24). 


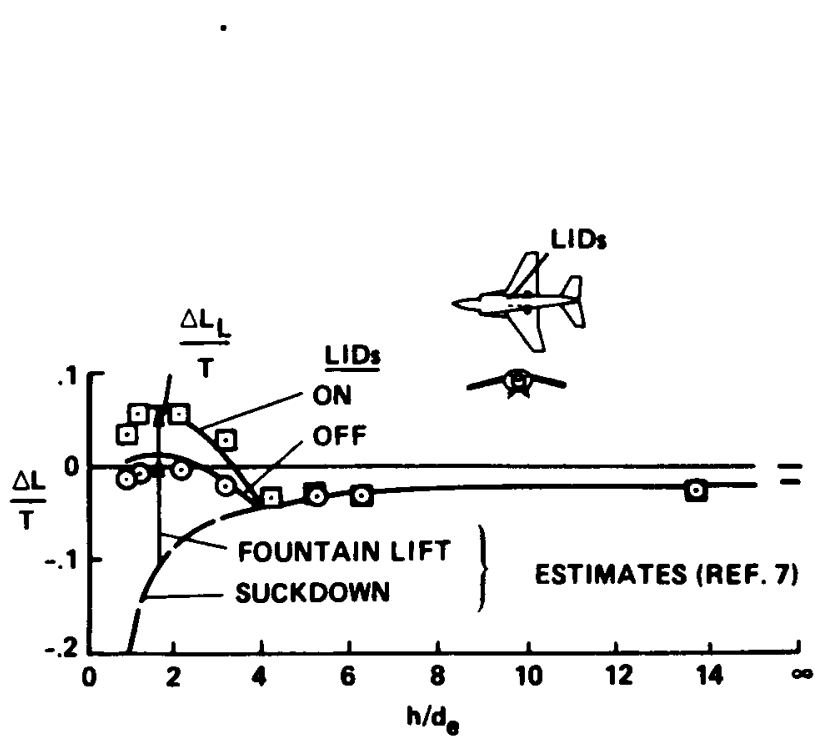

Fig. 8. Lift induced on a Harrier-type configuration hovering in ground effect (25).

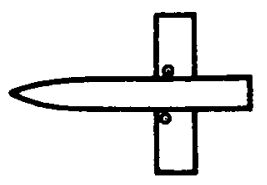

- eXPERIMENTAL DATA, (REF. 28)

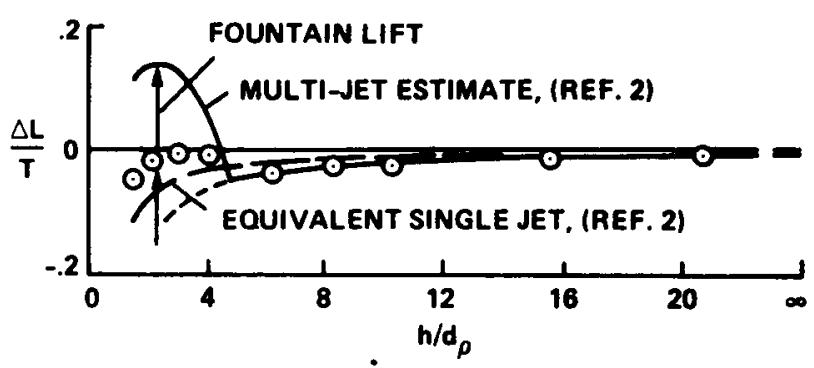

Fig. 9. Lift induced on a simple twin-jet highwing configuration hovering in ground effect (6).

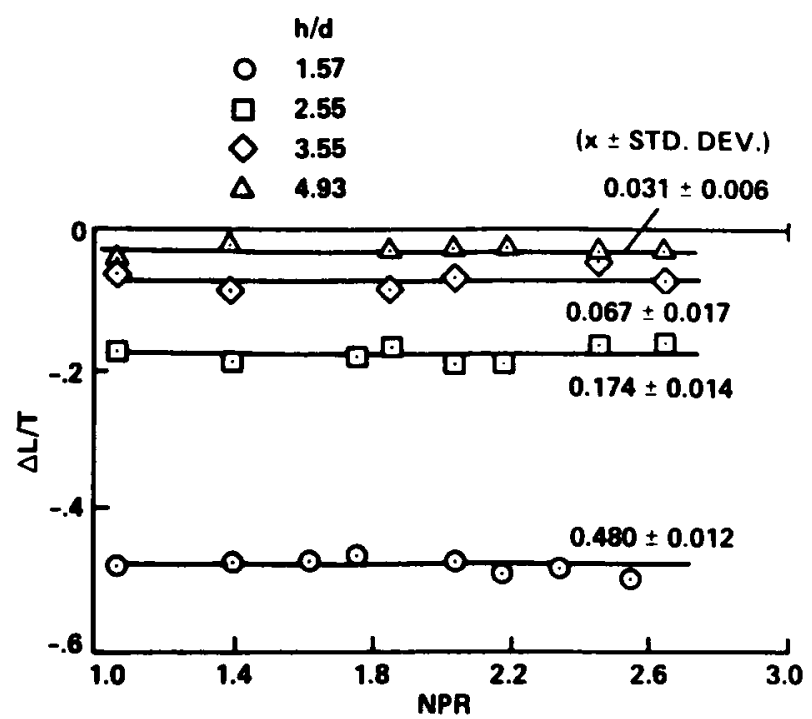

Fig. 10. Effect of nozzle pressure ratio on single-jet suckdown (27).

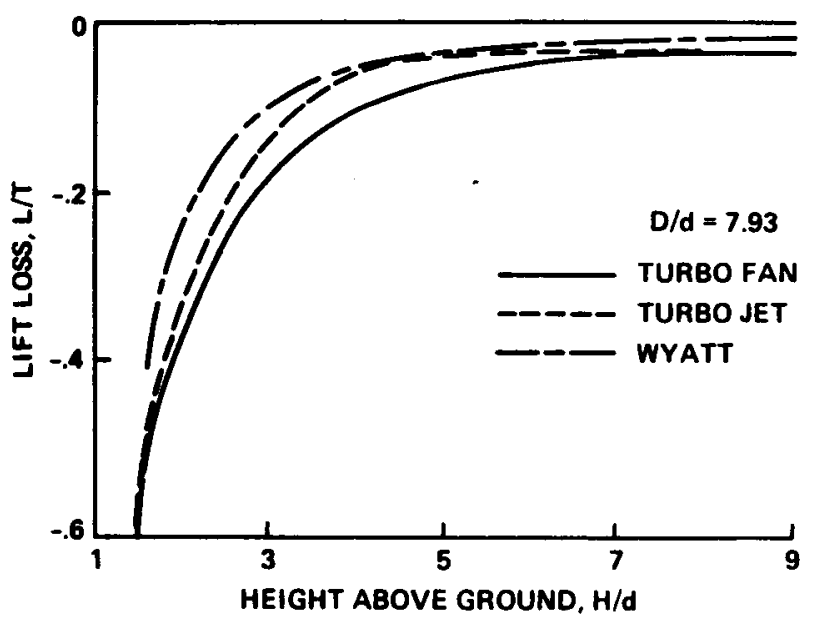

Eig. 11. Suckdown induced by full-scale jet engines (27) compared with Wyatt correction (20) based on small-scale data. 


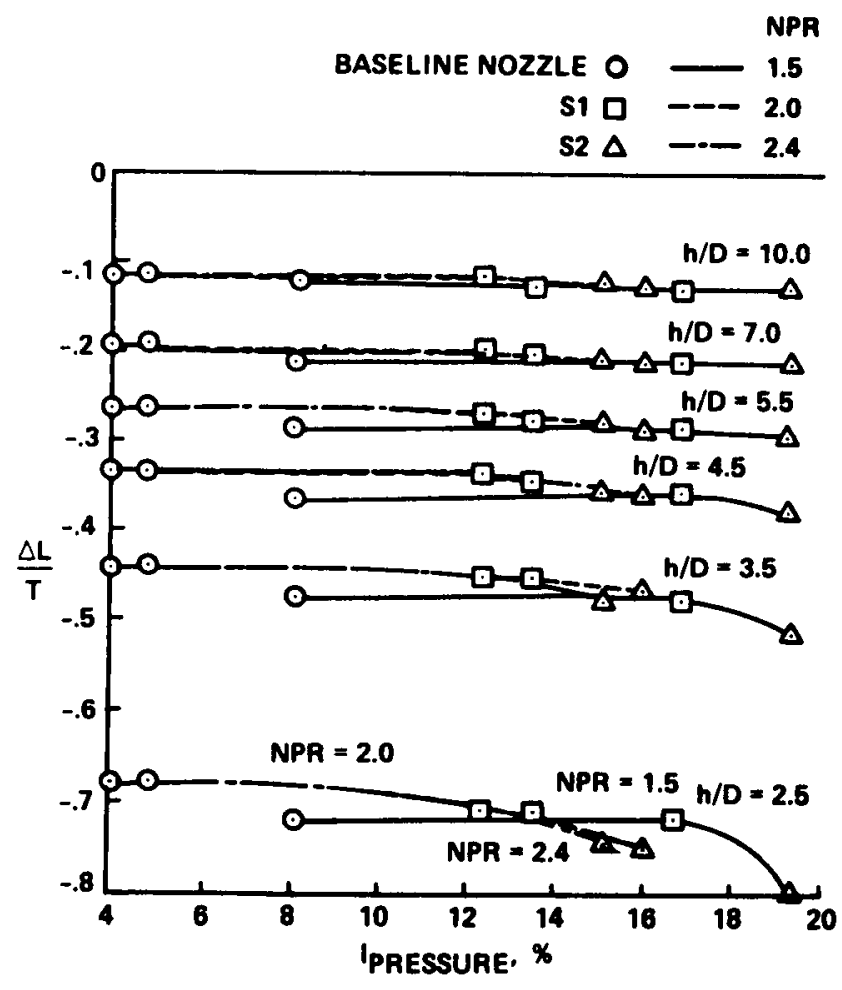

Eig. 12. Turbulence screen and nozzle pressure ratio effects on net induced lift for a twin-jet configuration (27).

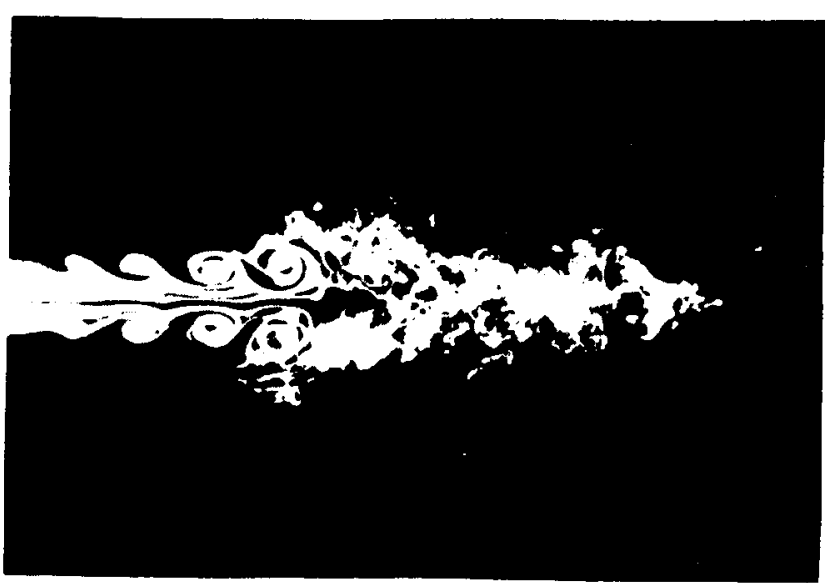

Fig. 13. Shadowgraph of jet $\left(R_{n}=10,000\right)$ showing initial laminar jet, the formation of ring vortices in the shear layer, and the transition to turbulence (28).

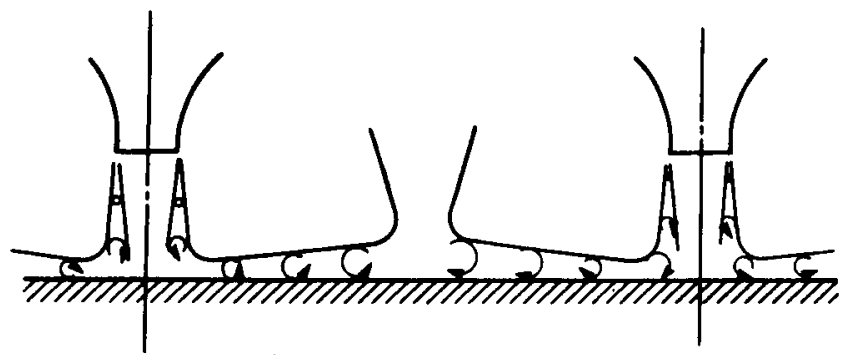

Fig. 14. Shear layer vortices may be the cause of fountain unsteadiness. 


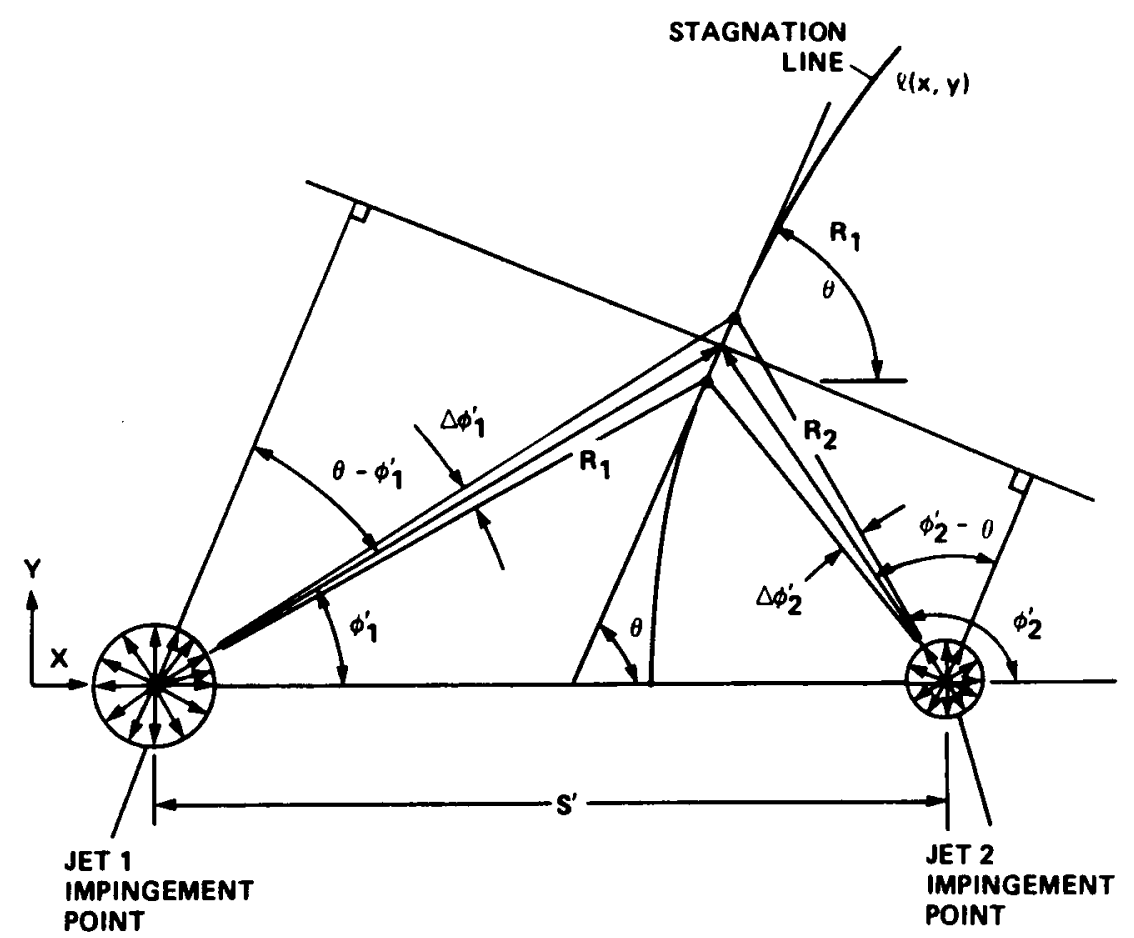

Eig. 15. A method for estimating the location and strength of the upwash fountain generated between impinging jets is presented in (2).

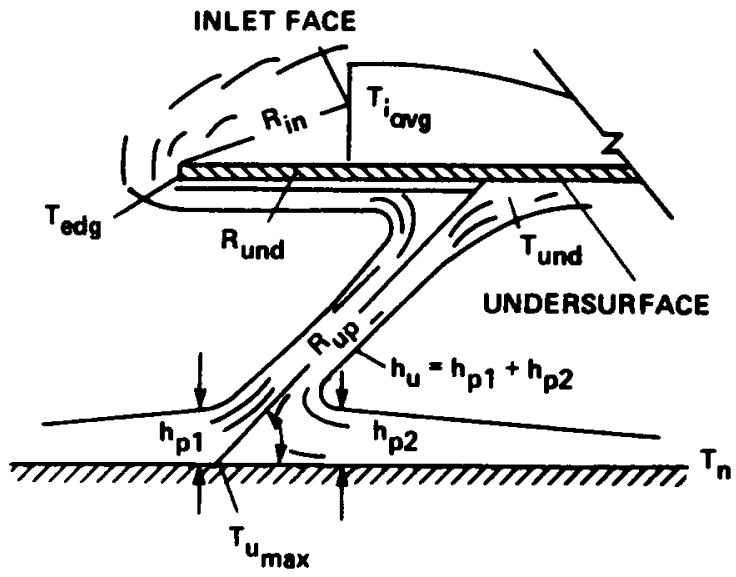

Fig. 16. A method for estimating the inlet temperature rise due to fountain flows by tracing the flow from impingement to the inlet is presented in (37).

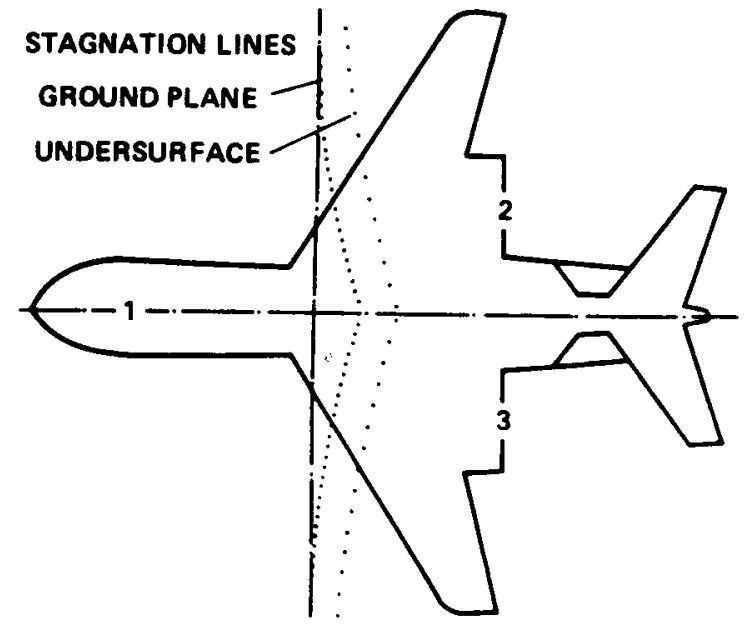

Fig. 17. Stagnation lines on the ground and on the aircraft lower surface calculated by the method of (37). 

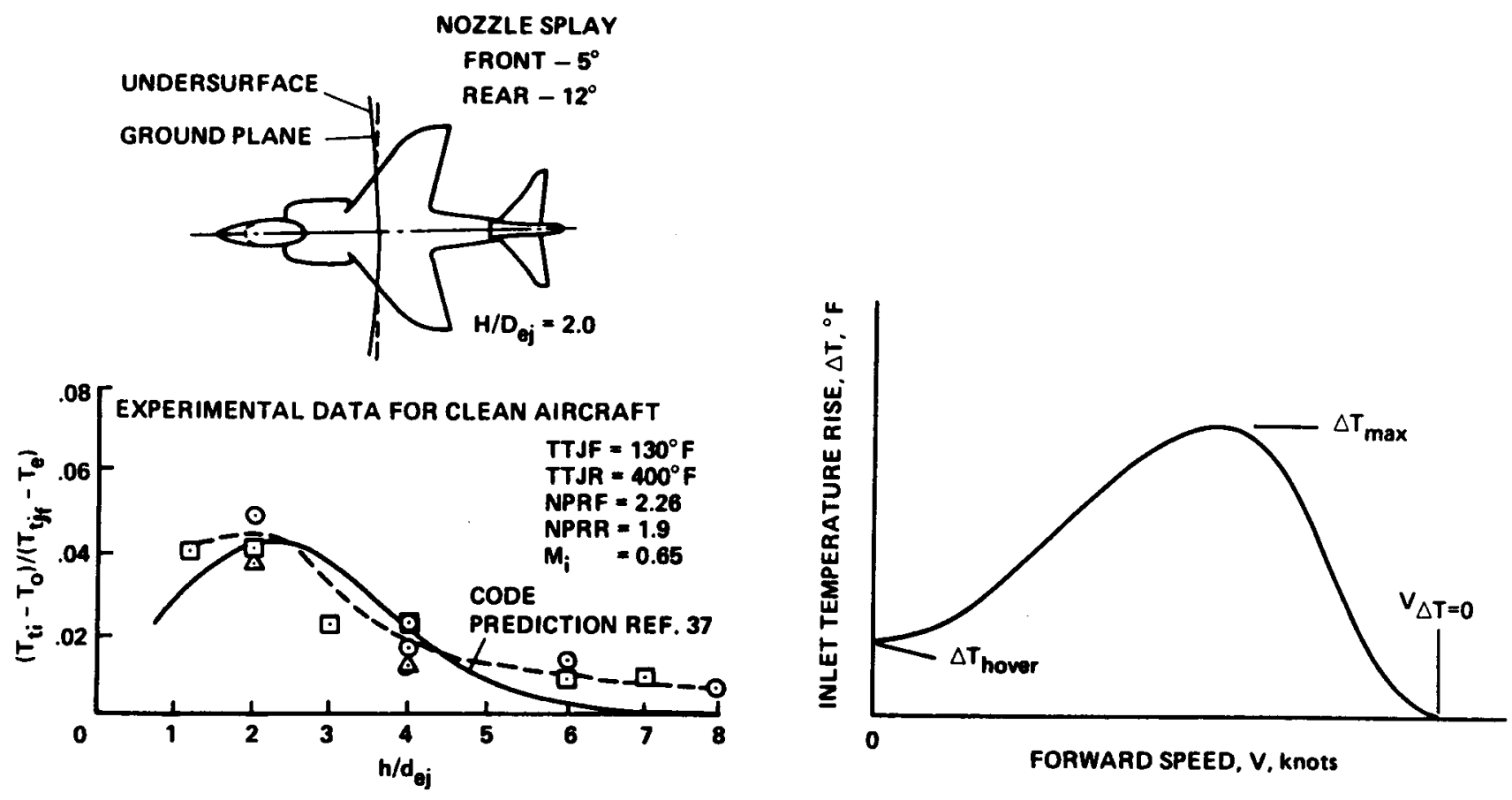

Fig. 18. Inlet temperature rise for a Harriertype aircraft estimated by the method of (37).

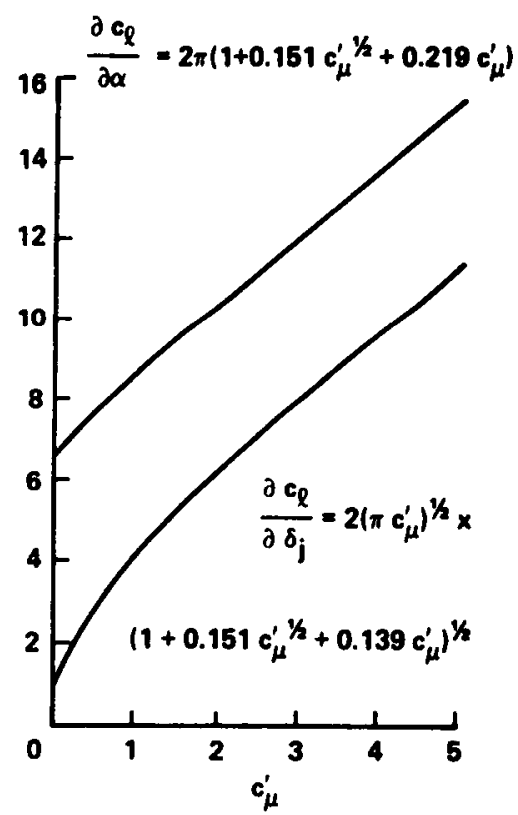

Fig. 19. Typical variation of inlet temperature rise with forward speed.
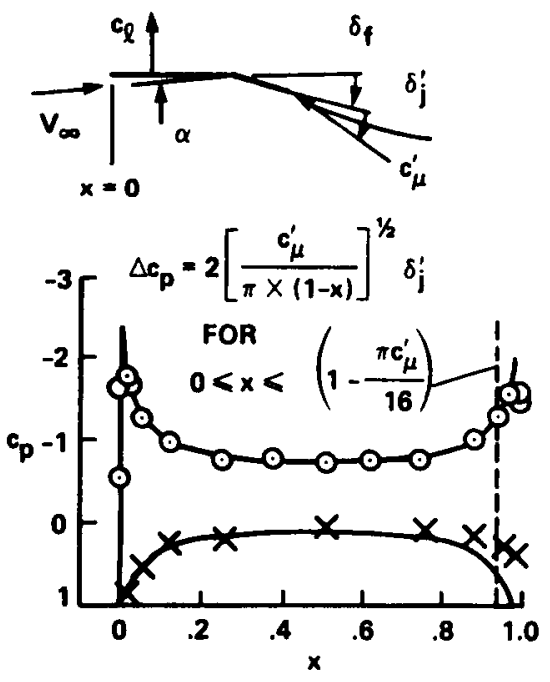

Fig. 20. Variation of 1 ift and pressure coefficients according to Spence's two-dimensional jet-flap theory (43). 


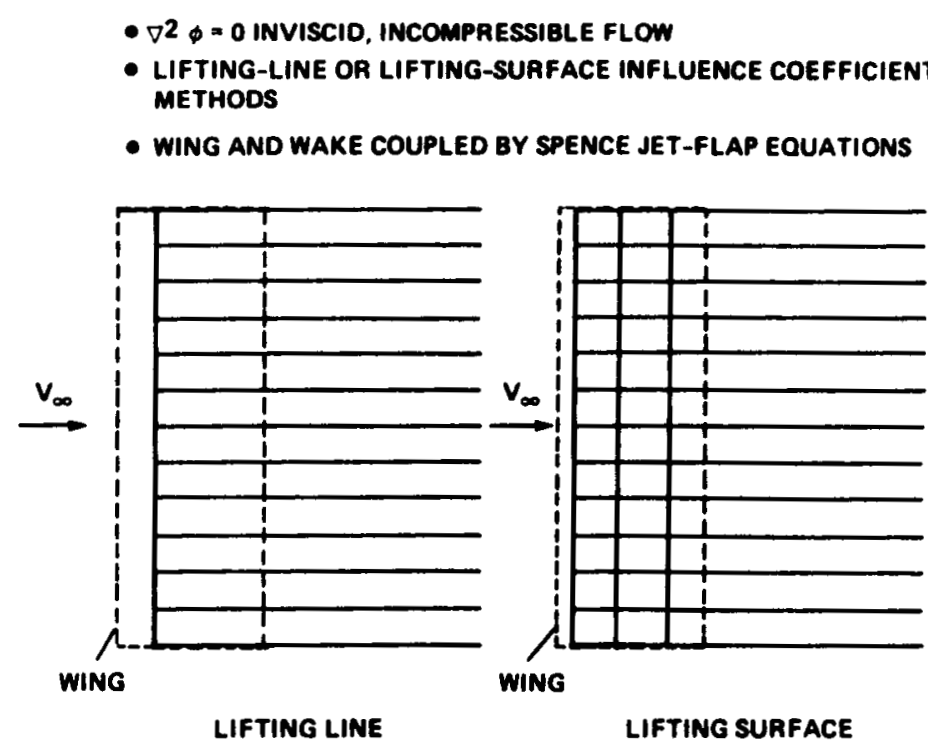

Fig. 21. Schematic of three-dimensional jet-flap wing lifting line and lifting surface methods.
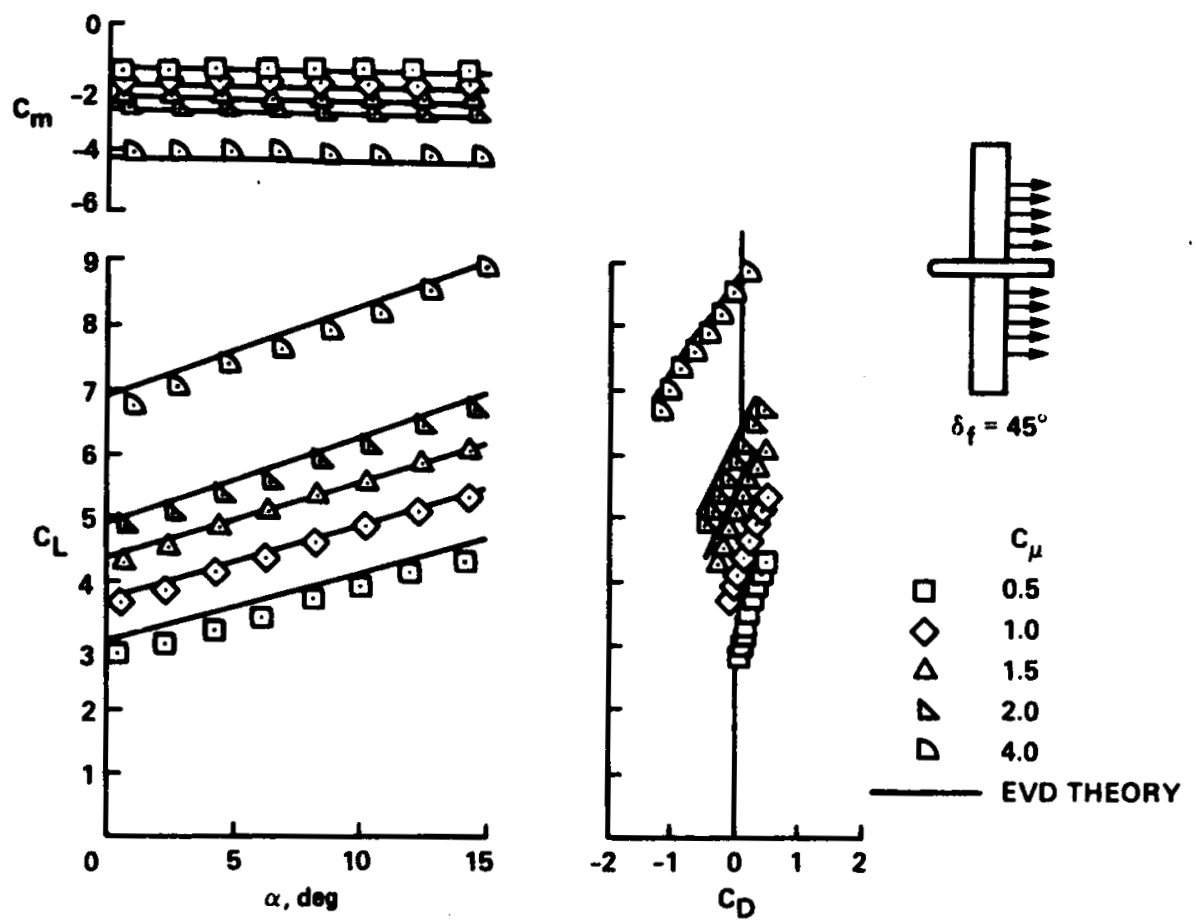

Fig. 22. Comparisons of EVD Jet-flap theory with experimental data for two-thirds span jetblown flap model. 

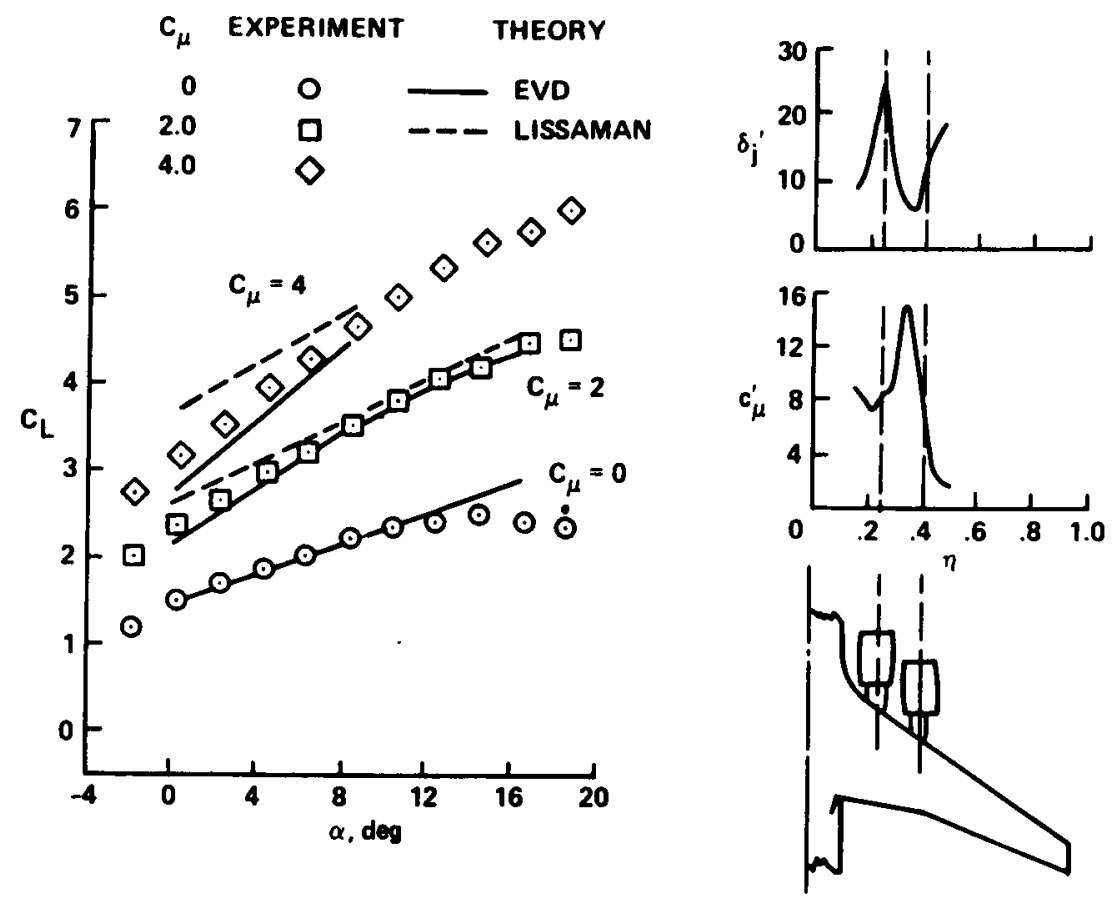

Fig. 23. Comparison of jet-flap theory with experimental data for an externally blown flap configuration.

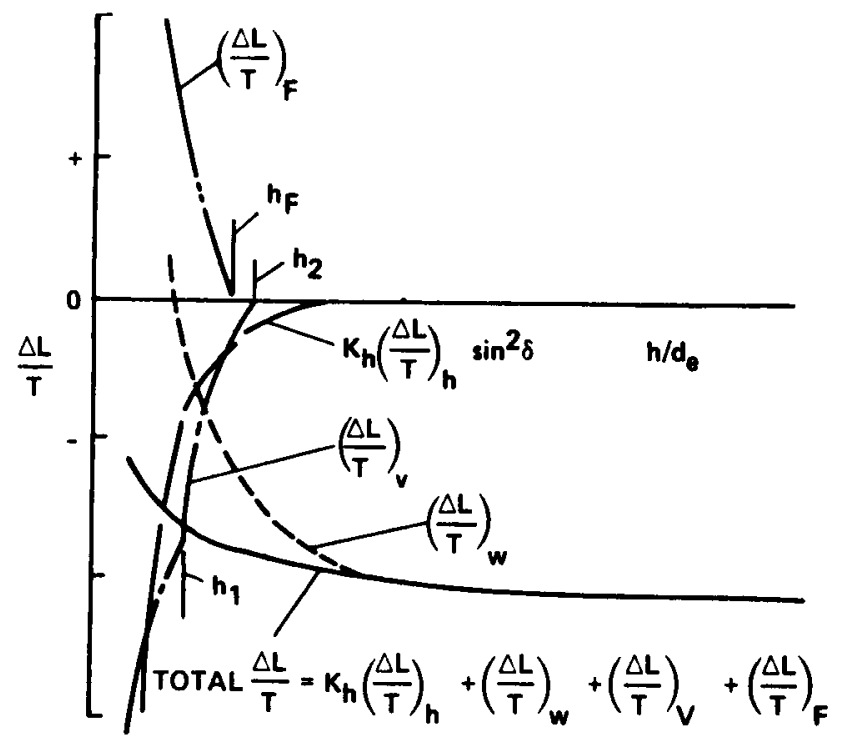

Eig. 24. Schematic of the effect of height on the induced $1 \mathrm{ift}$ increments in ground effect (26).

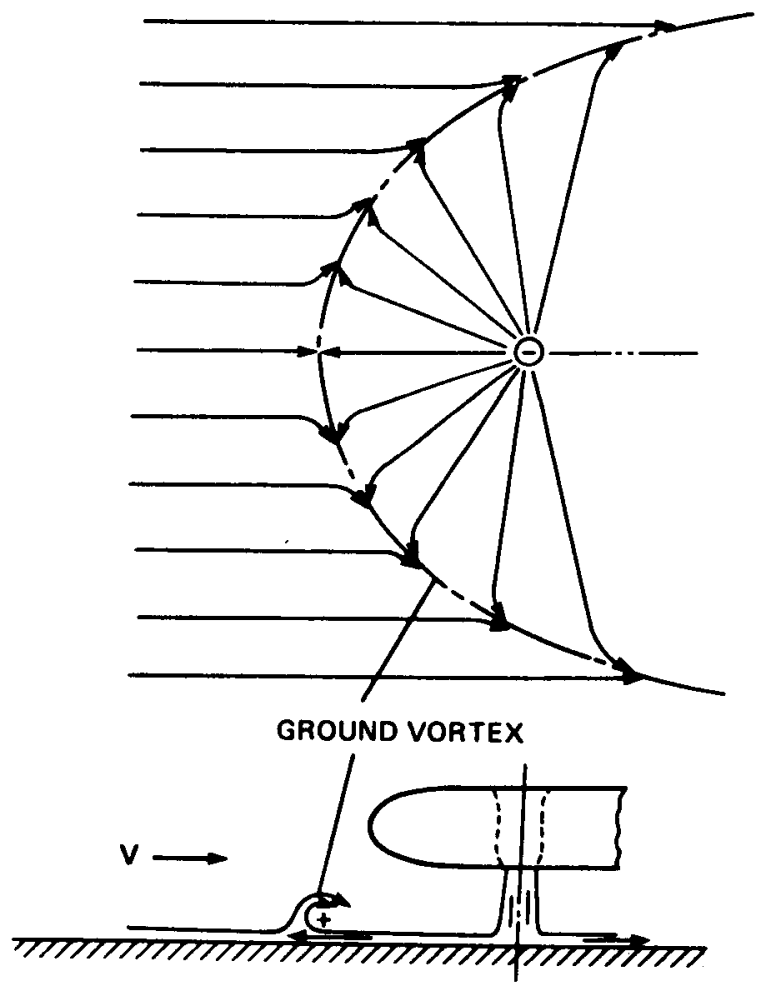

Fig. 25. Formation of the ground vortex. 


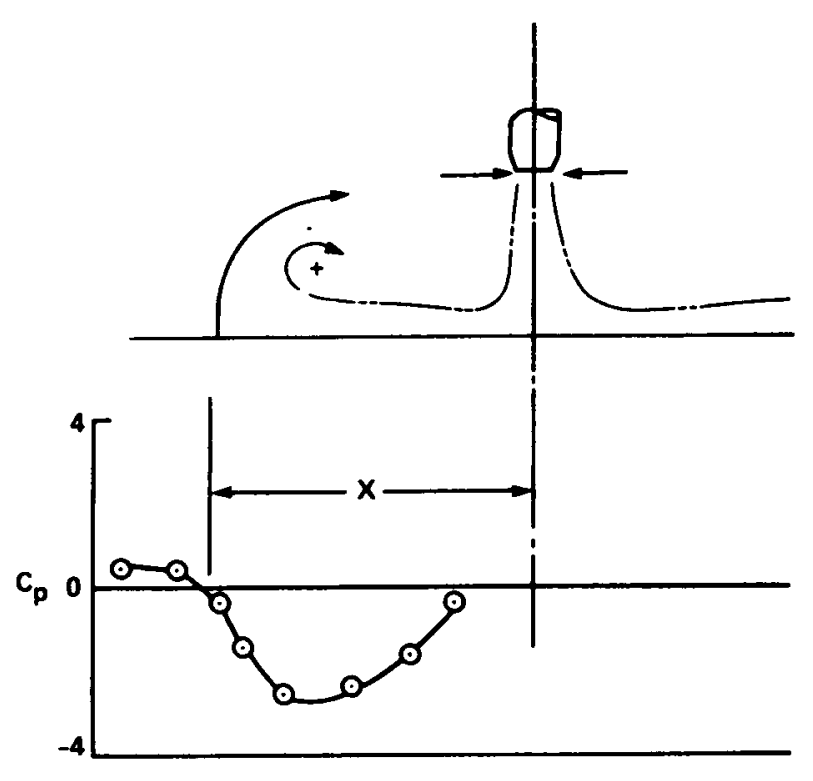

$$
-\frac{X}{D}=\frac{0.75}{v_{e}}-1.75\left(\frac{h}{d}\right)^{2.5} v_{e}^{2.25}
$$

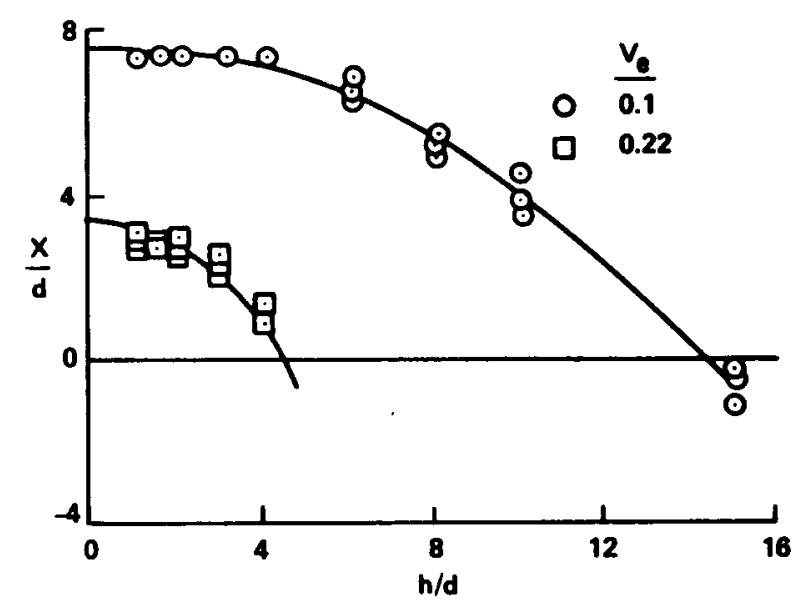

Fig. 26. Forward projection of the wall jet and zround vortex flow field (26).

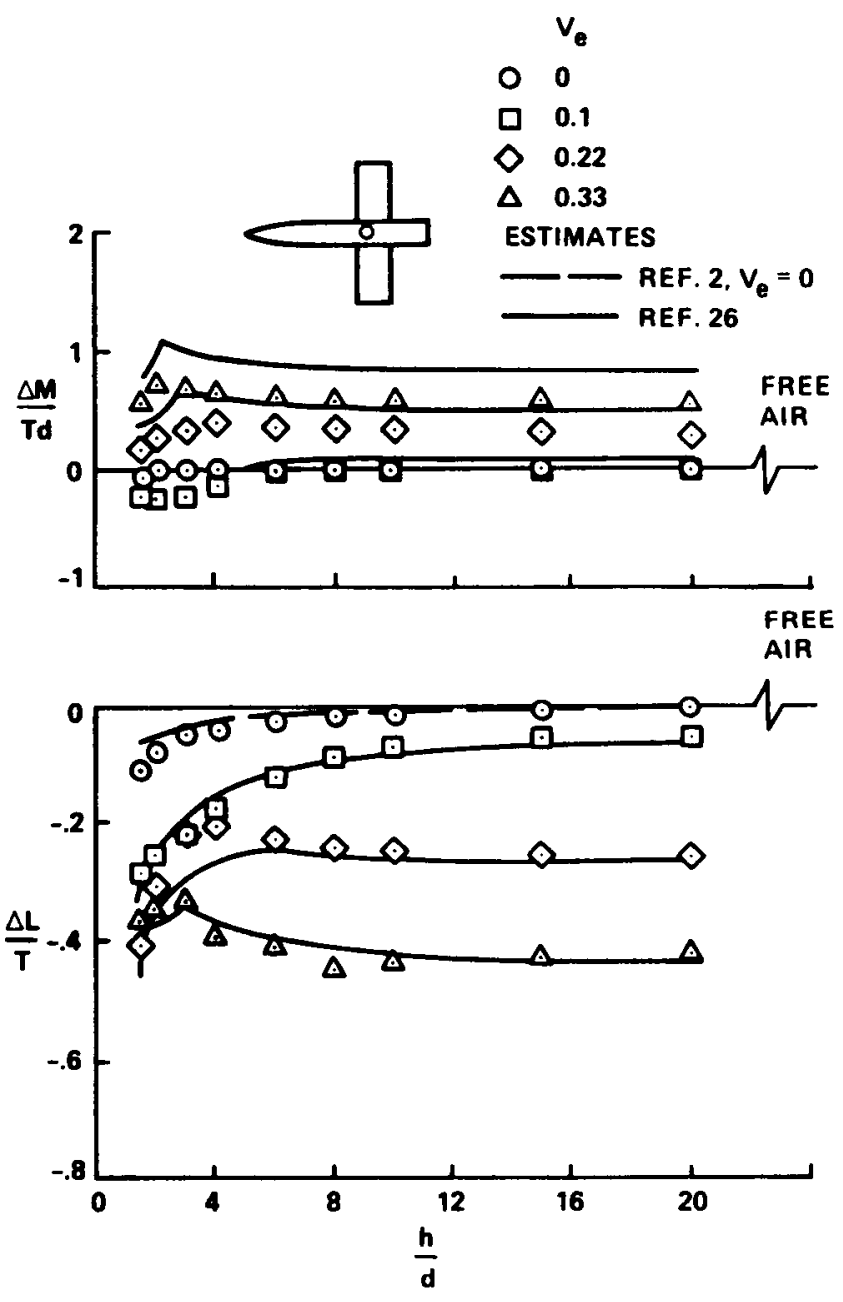

Fig. 27. Comparison of estimated ground effects with experimental data for a high-wing singlejet configuration from (26). 

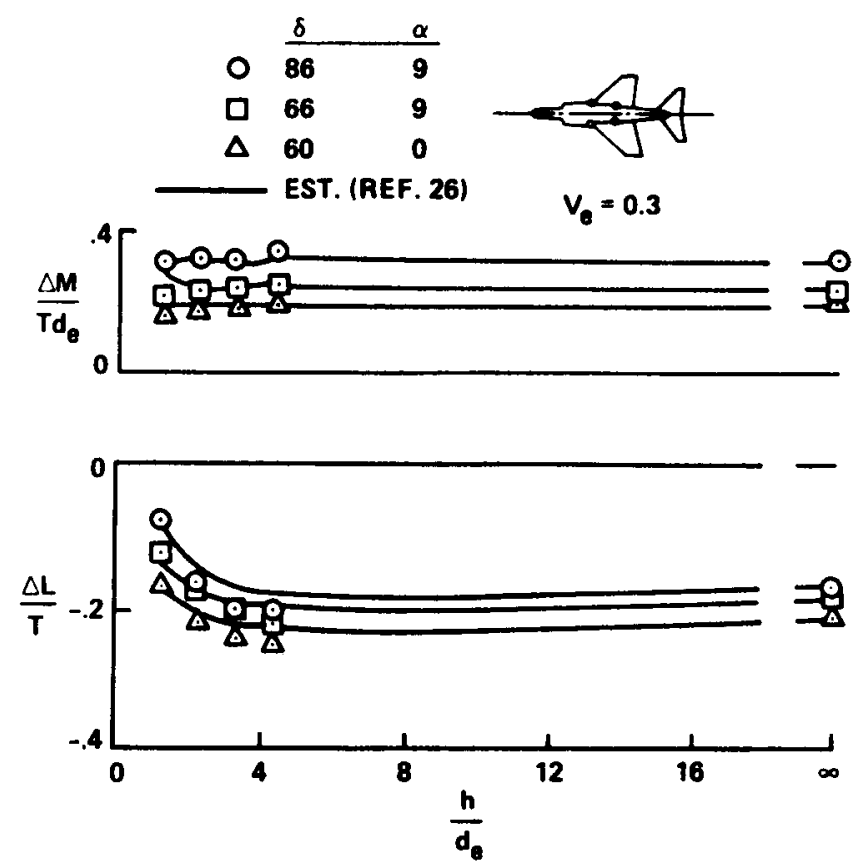

Eig. 28. Comparison of estimated ground effects with experimental data for a Harrier-type configuration of (53).
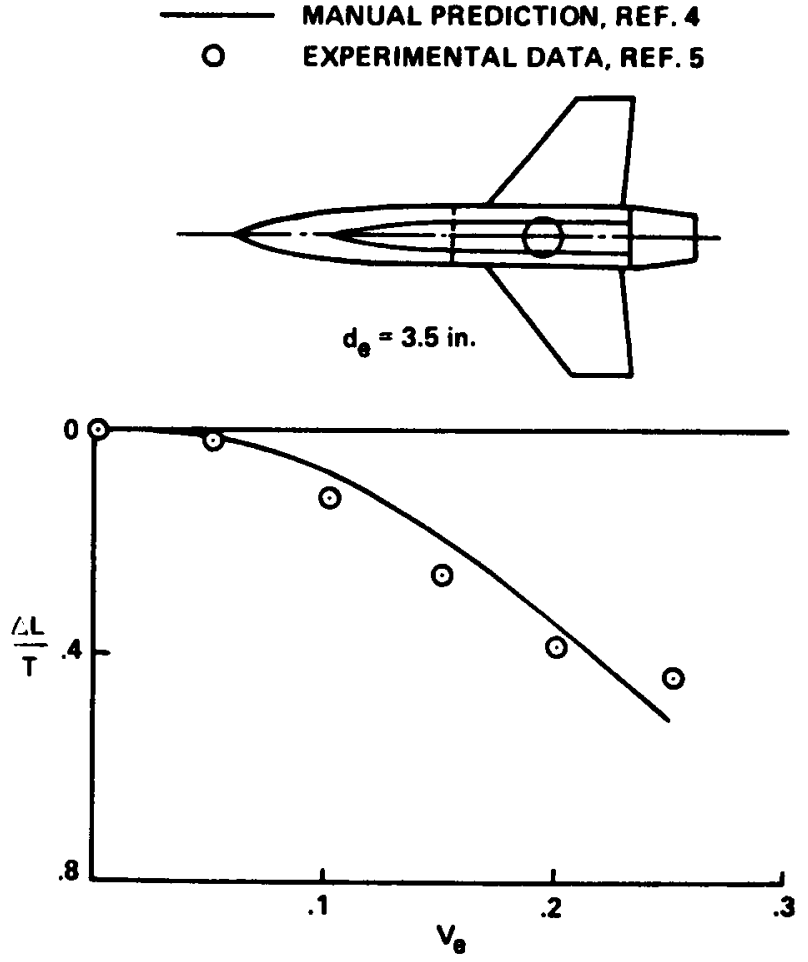

Fig. 29. Comparison of predicted and experimental data of clipped delta-wing configuration for a single-jet configuration, $\mathrm{Sj} / \mathrm{S}=0.024$
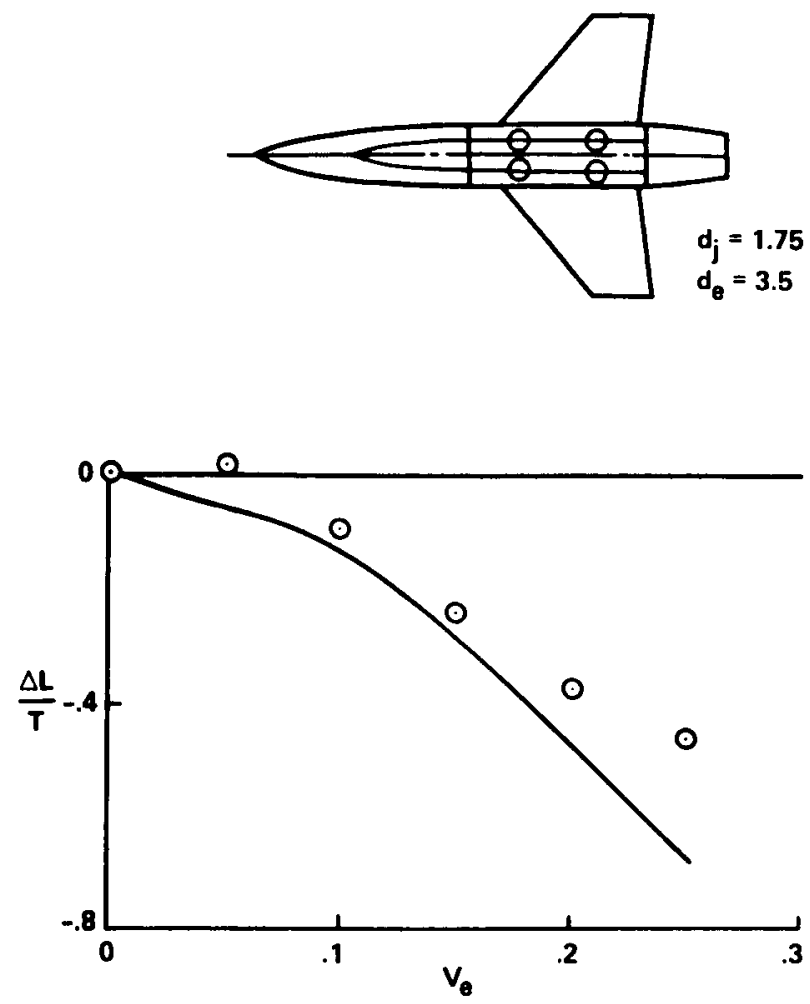

Fig. 30. Comparison of predicted and experimental data of clipped delta-wing configuration, $S J / S=0.024$. a) Single-jet configuration. b) Four-jet configuration. 

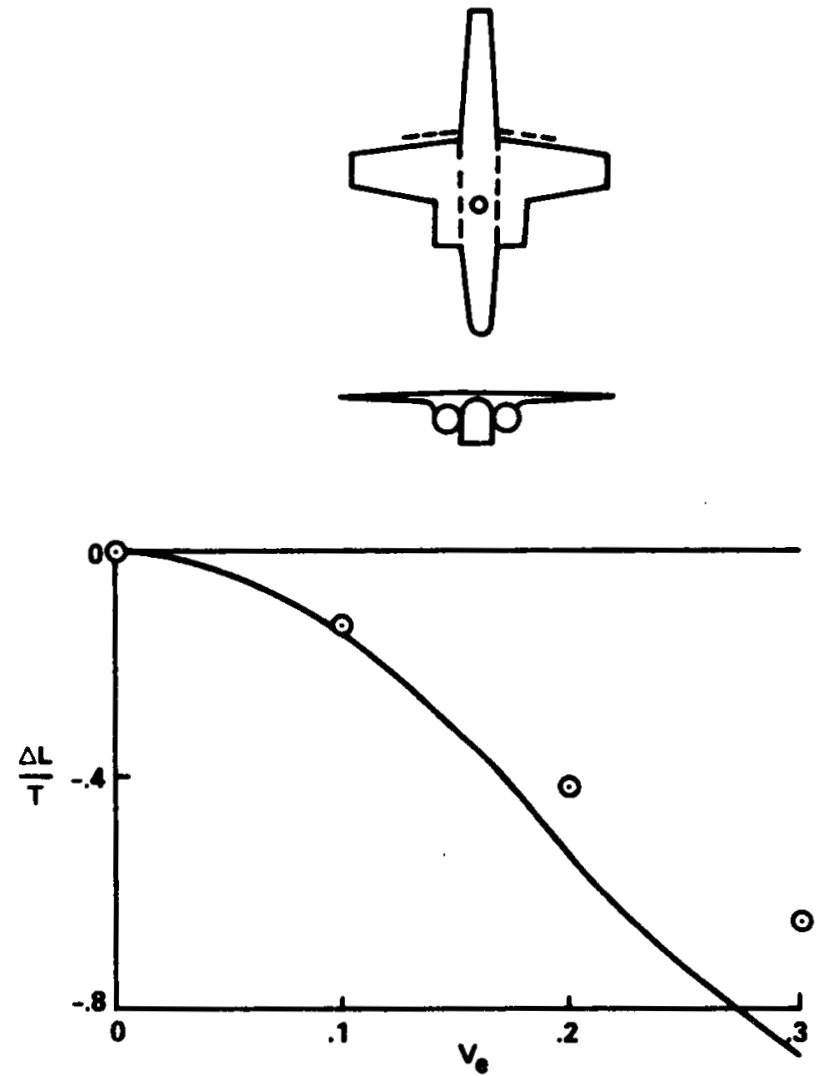

Fig. 31. Comparison of predicted and experimental data of a single-jet high-wing configuration. 

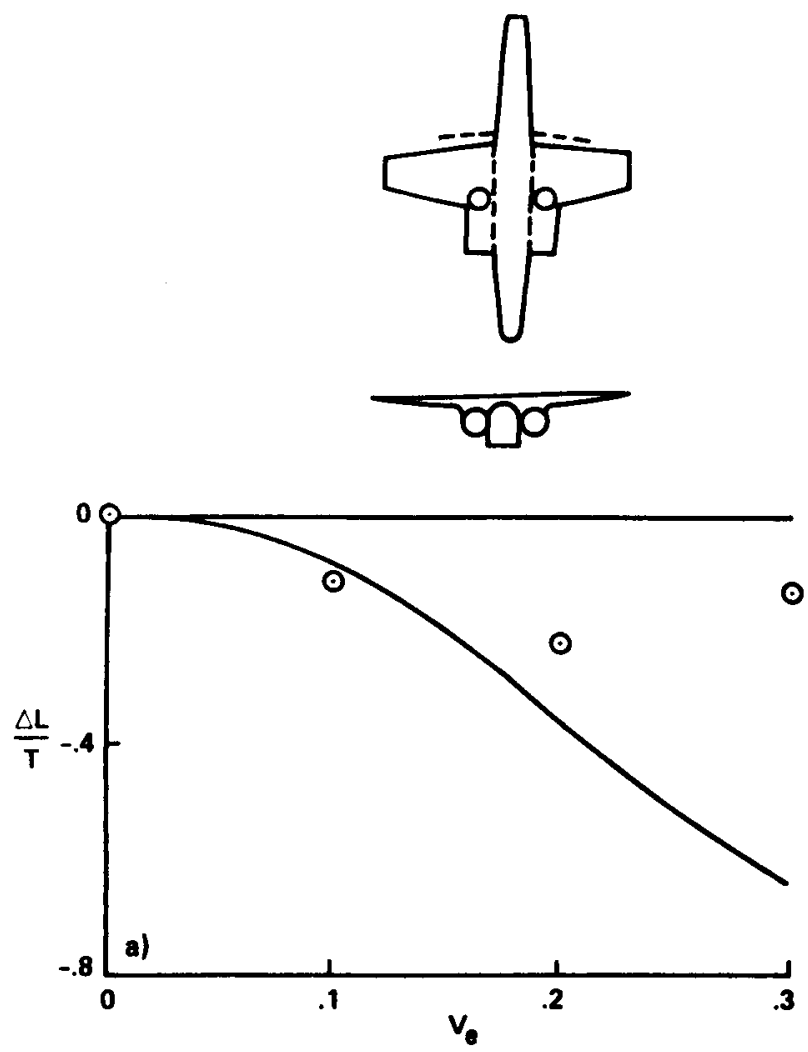
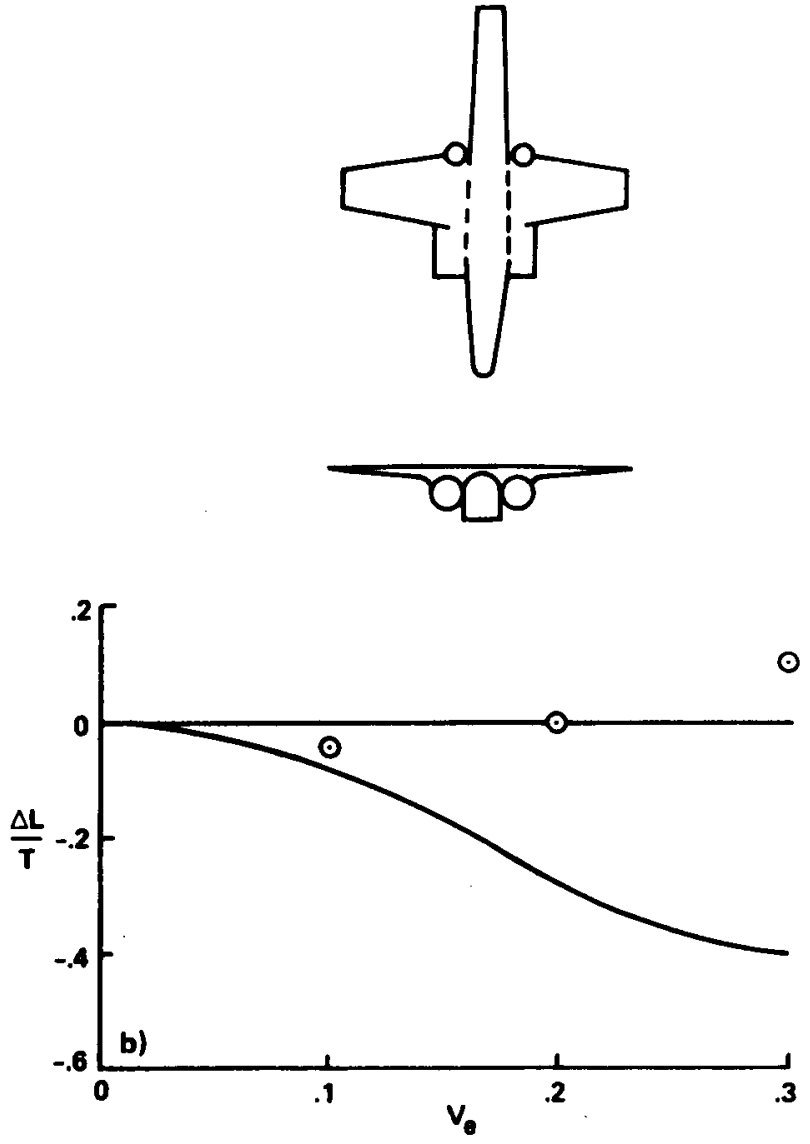

Fig. 32. Comparison of predicted and experimental data of a twin-jet, high-wing configuration. a) Nozzles in the forward position. b) Nozzles in the aft position.

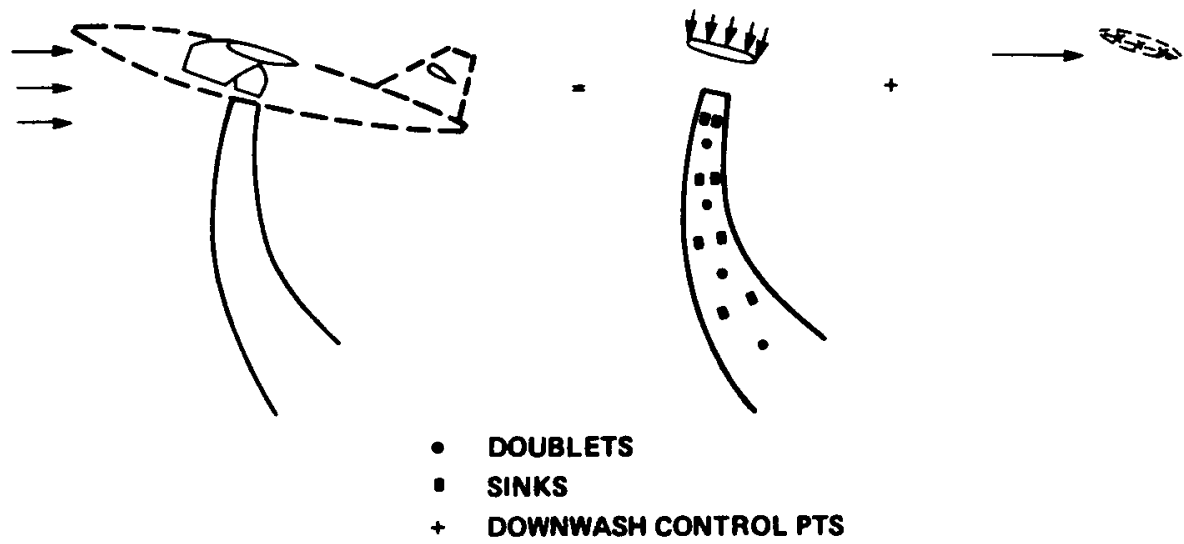

Eig. 33. Wooler method for computing the jet-induced effects on the wing using superposition of lifting-line wing and sink/doublet jet representations. 


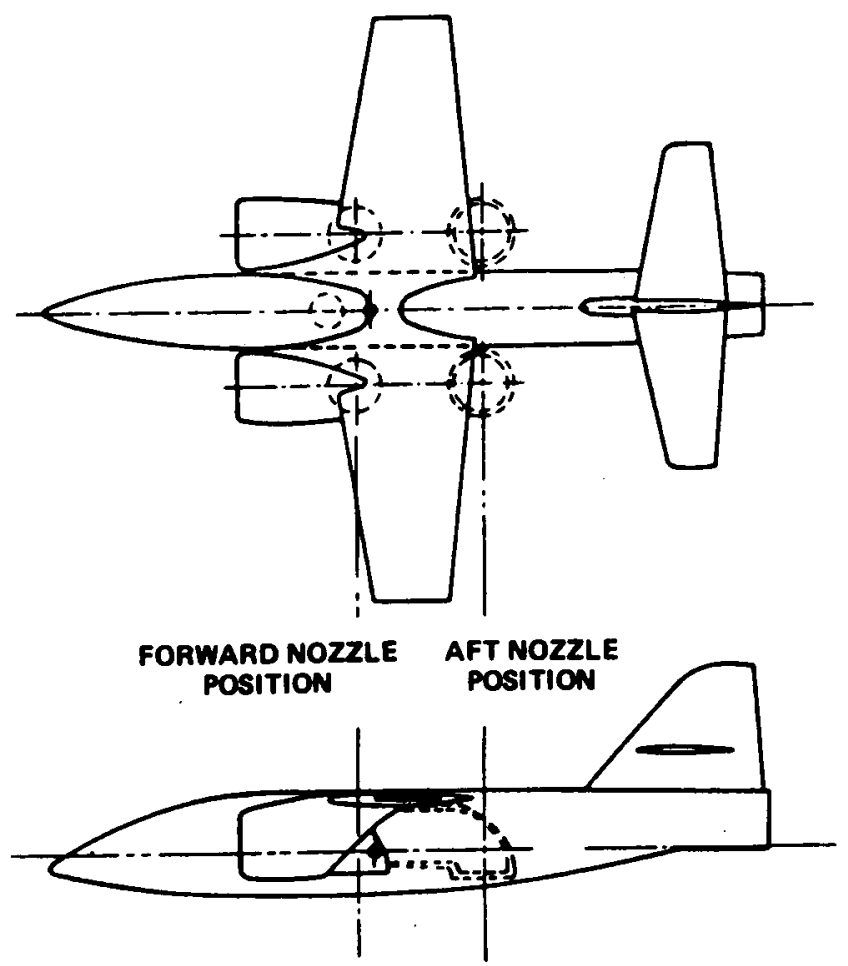

Fig. 34. Wind tunnel model of a subsonic VSTOL fighter concept (1,65 and 66).

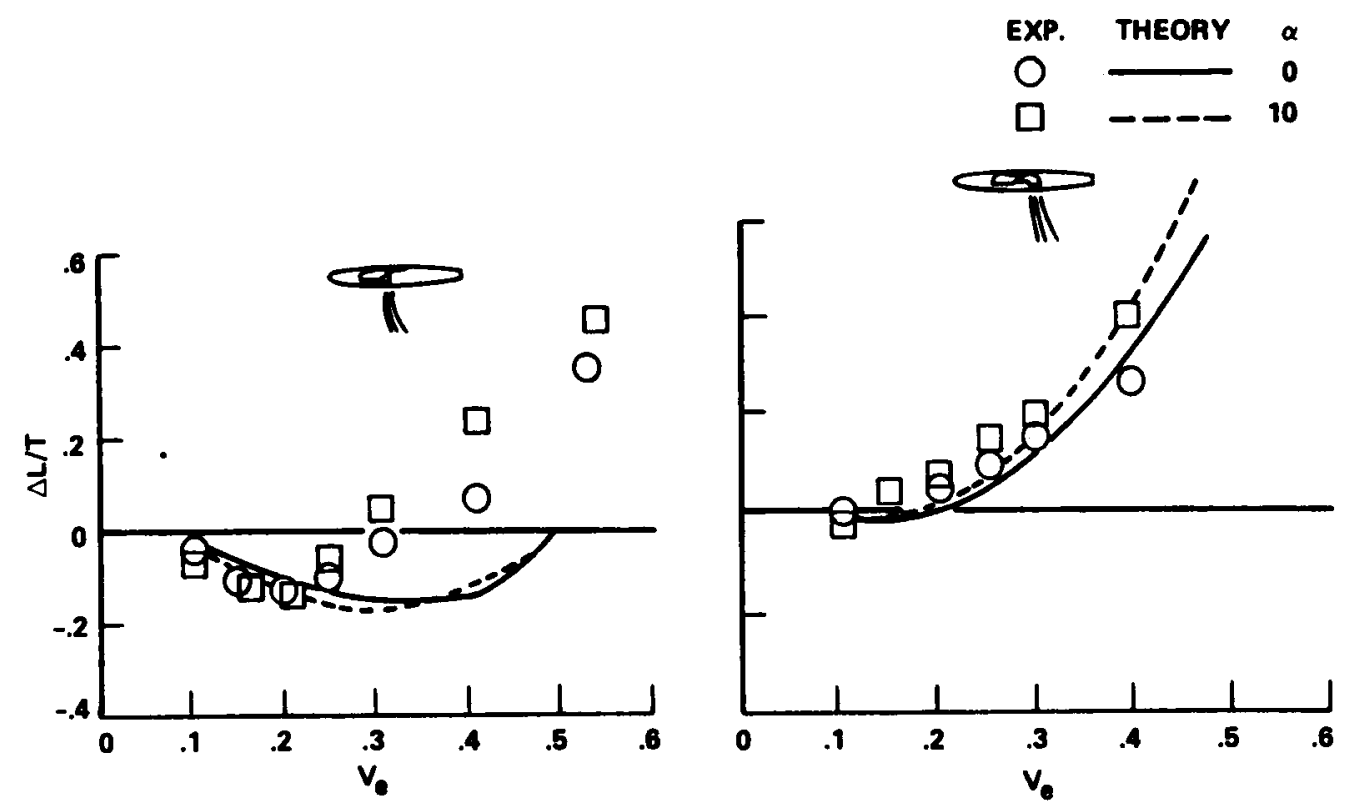

Fig. 35. Comparison between Wooler's original method and experimental data obtained on the model in Fig. 34. 


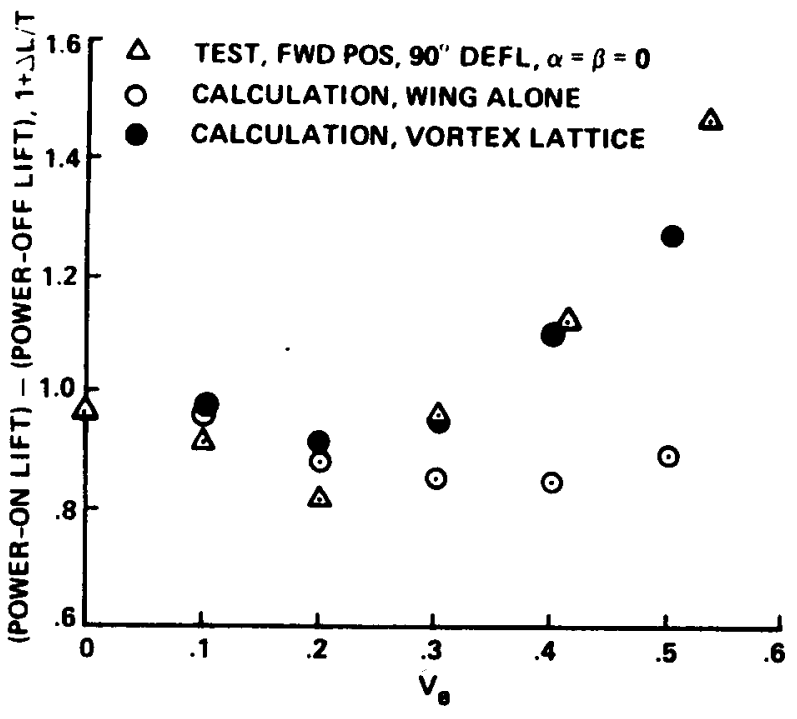

Fig. 36. Comparison among Wooler's original method (1), Wooler's improved method (59), and experimental data $(1,65$, and 66$)$ obtained on the model in Fig. 34 with the nozzles in the forward position.

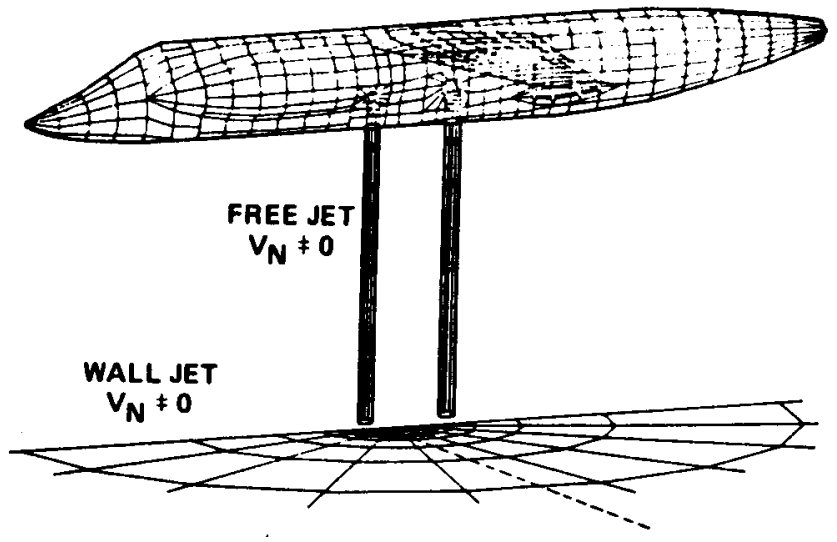

FIg. 37. Three-dimensional singularity-panel approach to VSTOL aircraft prediction methods (64).
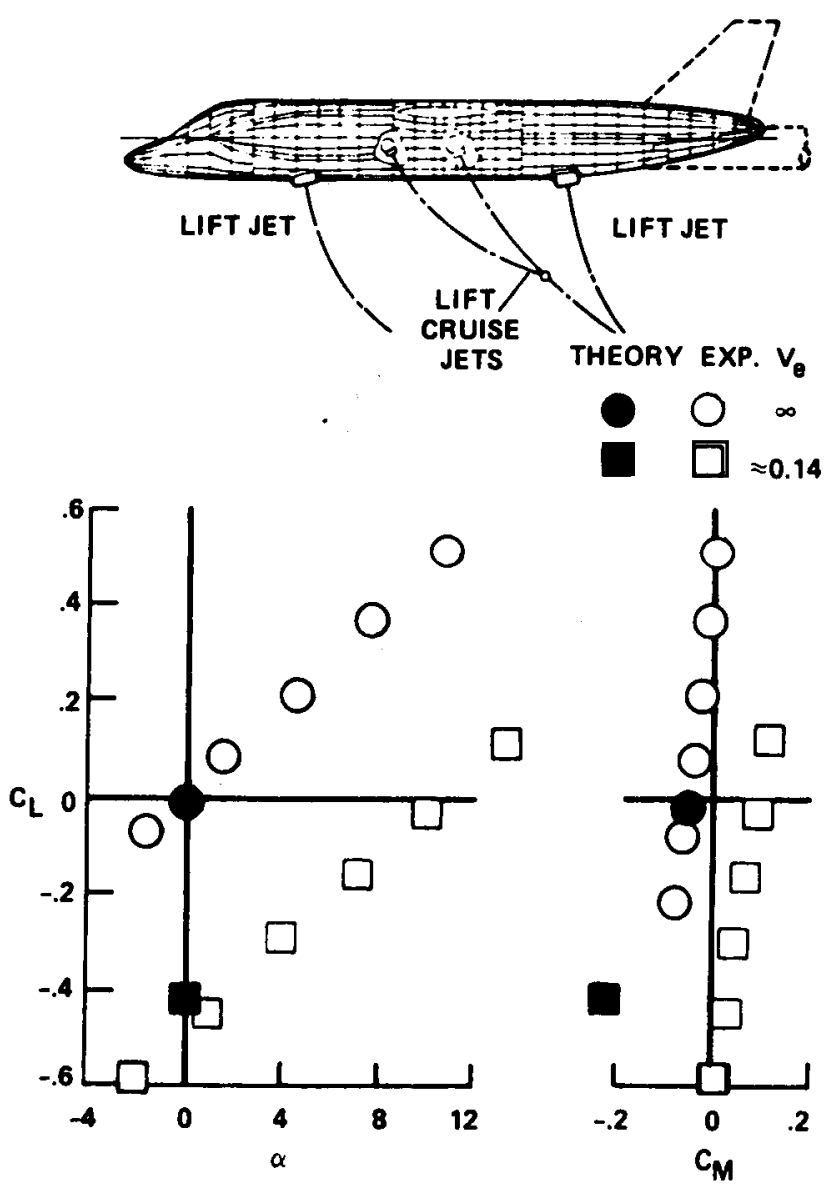

Fig. 38. Correlation between three-dimensional singularity-panel method and experimental data $(64)$. 

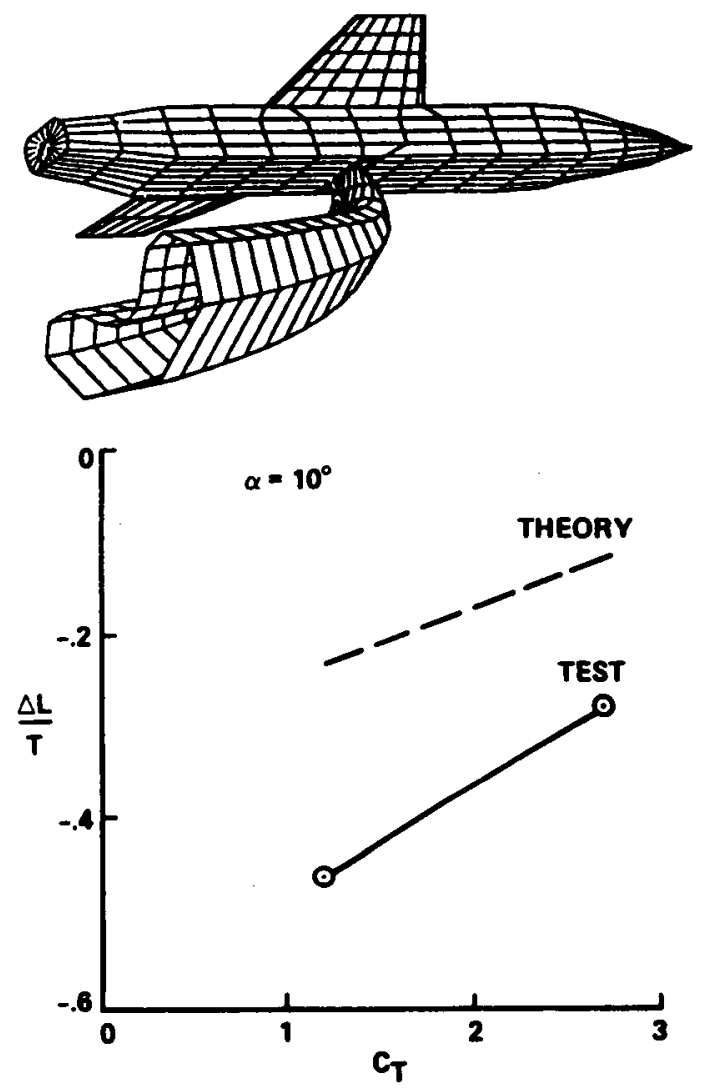

Fig. 39. Analysis of a VSTOL aircraft in transition flight using a PANAIR panel method and a parabolized jet wake model (69). 


\begin{tabular}{|c|c|c|c|c|}
\hline & & HOVER & TRANSITION & \\
\hline $\begin{array}{l}\text { OUT OF GROUND } \\
\text { EFFECT }\end{array}$ & $\begin{array}{l}\text { FORCES AND } \\
\text { MOMENTS }\end{array}$ & $Y^{17}{ }^{4}$ & & $\begin{array}{r}3 \\
4 \\
5 \\
6 \\
59 \\
60 \\
62 \\
72\end{array}$ \\
\hline \multirow[t]{3}{*}{$\begin{array}{c}\text { IN GROUND } \\
\text { EFFECT }\end{array}$} & $\begin{array}{l}\text { FORCES AND } \\
\text { MOMENTS }\end{array}$ & $\begin{array}{l}4 \\
6 \\
7\end{array}$ & & $\begin{array}{r}6 \\
26\end{array}$ \\
\hline & $\begin{array}{l}\text { HOT GAS } \\
\text { INGESTION }\end{array}$ & $\begin{array}{l}37 \\
38 \\
71\end{array}$ & & 39 \\
\hline & $\begin{array}{c}\text { GROUND } \\
\text { ENVIRONMENT }\end{array}$ & $\begin{array}{r}2 \\
37\end{array}$ & & $\begin{array}{l}26 \\
29 \\
73\end{array}$ \\
\hline
\end{tabular}

Fig. 40. Adequacy of available prediction methods; shaded area indicates percent of need covered; numbers indicate key references. 


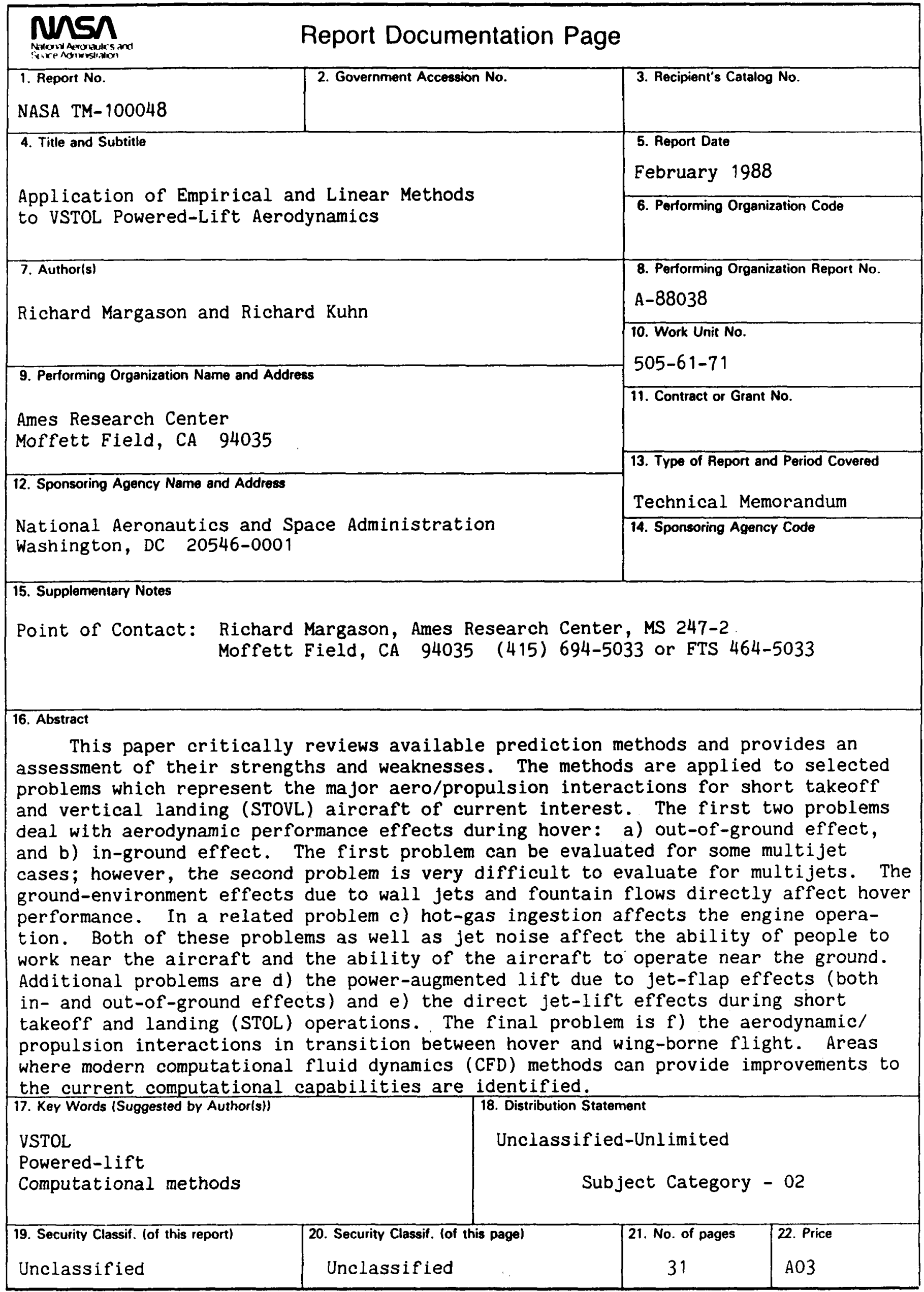

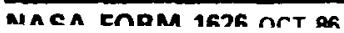

